\title{
Possible superconductivity from incoherent carriers in overdoped cuprates
}

M. Čulo ${ }^{1}{ }^{\dagger}$ C. Duffy ${ }^{\dagger 1}$, J. Ayres ${ }^{1,2}$, M. Berben ${ }^{1}$, Y.-T. Hsu ${ }^{1}$, R. D. H. Hinlopen ${ }^{2}$, B. Bernáth $^{1}$, N. E. Hussey ${ }^{1,2}$

1 High Field Magnet Laboratory (HFML-EMFL) and Institute for Molecules and Materials, Radboud University, Toernooiveld 7, 6525 ED Nijmegen, Netherlands 2 H. H. Wills Physics Laboratory, University of Bristol, Tyndall Avenue, Bristol BS8 1TL, United Kingdom *matija.culo@ru.nl, caitlin.duffy@ru.nl, n.e.hussey@bristol.ac.uk

February 21, 2021

\begin{abstract}
The non-superconducting state of overdoped cuprates is conjectured to be a strange metal comprising two distinct charge sectors, one governed by coherent quasiparticle excitations, the other seemingly incoherent and characterized by non-quasiparticle (Planckian) dissipation. The zero-temperature superfluid density $n_{s}(0)$ of overdoped cuprates exhibits an anomalous depletion with increased hole doping $p$, falling to zero at the edge of the superconducting dome. Over the same doping range, the effective zero-temperature Hall number $n_{\mathrm{H}}(0)$ transitions from $p$ to $\mathbf{1}+p$. By taking into account the presence of these two charge sectors, we demonstrate that in the overdoped cuprates $\mathbf{T l}_{2} \mathbf{B a}_{2} \mathbf{C u O}_{6+\delta}$ and $\mathrm{La}_{2-x} \mathrm{Sr}_{x} \mathrm{CuO}_{4}$, the growth in $n_{s}(0)$ as $p$ is decreased from the overdoped side may be compensated by the loss of carriers in the coherent sector. Such a correspondence is contrary to expectations from conventional BCS theory and implies that superconductivity in overdoped cuprates emerges uniquely from the sector that exhibits incoherent transport in the normal state.
\end{abstract}

\section{Contents}

\begin{tabular}{lll}
\hline & Introduction & 2
\end{tabular}

2 Superfluid and coherent carrier density in $\mathrm{Tl}_{2} \mathrm{Ba}_{2} \mathrm{CuO}_{6+\delta} \quad 3$

3 Superfluid and coherent carrier density in $\mathrm{La}_{2-x} \mathrm{Sr}_{x} \mathrm{CuO}_{4} \quad 5$

\begin{tabular}{lll}
\hline 4 & Discussion & 9
\end{tabular}

$\begin{array}{lll}5 \text { Conclusion } & 11\end{array}$

\begin{tabular}{|ll|}
\hline A Obtaining estimates for $n_{c o h}$ and $n_{s}(0)$ in $\mathbf{T l}_{2} \mathbf{B a}_{2} \mathbf{C u O}_{6+\delta}$ & $\mathbf{1 1}$
\end{tabular}

$\begin{array}{lll}\text { A.1 Additive conductivity channels in } \mathrm{Tl}_{2} \mathrm{Ba}_{2} \mathrm{CuO}_{6+\delta} & 12\end{array}$

\begin{tabular}{lll}
\hline A.2 & Fitting the zero-field resistivity & 14
\end{tabular}

\begin{tabular}{lll}
\hline \hline A.3 & Coherent carrier density in $\mathrm{Tl}_{2} \mathrm{Ba}_{2} \mathrm{CuO}_{6+\delta}$ & 16
\end{tabular}

\begin{tabular}{|lll}
\hline \hline A.4 & Planckian dissipation in $\mathrm{Tl}_{2} \mathrm{Ba}_{2} \mathrm{CuO}_{6+\delta}$ & 17
\end{tabular}

${ }^{\dagger}$ These authors contributed equally to this work 
\begin{tabular}{|ll|ll}
\hline A.5 Validity of approximations used in Section & A.1 & 17
\end{tabular}

\begin{tabular}{lll}
\hline \hline A.6 & Superfluid density in $\mathrm{Tl}_{2} \mathrm{Ba}_{2} \mathrm{CuO}_{6+\delta}$ & 18
\end{tabular}

B Obtaining estimates for $n_{c o h}$ and $n_{s}(0)$ in $\mathbf{L a}_{2-x} \mathbf{S r}_{x} \mathbf{C u O}_{4} \quad 19$

\begin{tabular}{lll}
\hline B.1 Hall effect in $\mathrm{La}_{2-x} \mathrm{Sr}_{x} \mathrm{CuO}_{4}$ & 19
\end{tabular}

B.2 Estimating the superfluid density in $\mathrm{La}_{2-x} \mathrm{Sr}_{x} \mathrm{CuO}_{4}$

\begin{tabular}{lll}
\hline \hline B.3 & Coherent carrier density in $\mathrm{La}_{2-x} \mathrm{Sr}_{x} \mathrm{CuO}_{4}$ & 24
\end{tabular}

\begin{tabular}{lll}
\hline B.4 & The weighting factor $f_{\sigma}$ in $\mathrm{La}_{2-x} \mathrm{Sr}_{x} \mathrm{CuO}_{4}$ & 26
\end{tabular}

\begin{tabular}{lll}
\hline B.5 & Additional considerations for $\mathrm{La}_{2-x} \mathrm{Sr}_{x} \mathrm{CuO}_{4}$ & 27
\end{tabular}

\begin{tabular}{|lll} 
C Dirty $d$-wave scenario in overdoped cuprates revisited & 28
\end{tabular}

\begin{tabular}{lr}
\hline References & 30 \\
\hline
\end{tabular}

\section{Introduction}

As in many other unconventional superconductors, the transition temperature $T_{c}$ of high$T_{c}$ cuprates traces out a dome in their phase diagram. In cuprates, this dome is parameterised by a range of doping $p$ (across which superconductivity appears) and a maximum $T_{c}$ at optimal (OP) doping. The reasons for the loss of superconductivity on either side of the dome are not yet well understood, though it is generally believed that on the underdoped (UD) side, proximity to the Mott insulating state is key [1], while on the overdoped (OD) side, superconductivity vanishes due to a diminishing pairing interaction [2,3]. The anomalously low superfluid density $n_{s}(0)$ found early on in OD cuprates [4, 5] was then attributed to pair breaking, following standard BCS treatments for a disordered or 'dirty' $d$-wave superconductor 6 .

A challenge to this viewpoint emerged in a recent study of the superfluid density in OD $\mathrm{La}_{2-x} \mathrm{Sr}_{x} \mathrm{CuO}_{4}$ (LSCO) thin films 7]. There, the decrease in $n_{s}(0)$ on approach to the edge of the superconducting $(\mathrm{SC})$ dome at $p_{S C} \sim 0.27$ was mapped out in great detail. The authors argued that while pair-breaking due to disorder can reduce $n_{s}(0)$, the levels of pair-breaking required within conventional BCS theory would render the $T$-dependence of the superfluid density $n_{s}(T)$ quadratic over a wide temperature range, in contrast to the observed $T$-linear behavior. Its explanation, the authors concluded [7], lay outside the realms of BCS theory.

The key requisite of a BCS superconductor is a Fermi-liquid (FL) ground state with a well-defined Fermi surface (FS). In OD $\mathrm{Tl}_{2} \mathrm{Ba}_{2} \mathrm{CuO}_{6+\delta}$ (Tl2201) with $T_{c}<30 \mathrm{~K}(p>$ 0.27 ), the observation of quantum oscillations (QO) appeared to confirm the existence of a large hole-like FS containing $1+p$ carriers (corresponding to the full Luttinger count) 8]. As such, OD cuprates are commonly perceived to be conventional, both in their normal and superconducting states [9]. In reality, the normal state transport properties of all OD cuprates, including T12201, are far from conventional. This so-called 'strange metal' regime has three notable characteristics: (i) a ubiquitous non-FL ( $T$-linear) component in the inplane resistivity $\rho_{a b}(T)$ at low $T$ 10 12], whose coefficient $\alpha(0)$ scales with $T_{c}$ 12, 13]; (ii) a Hall number $n_{\mathrm{H}}(0)$ deduced from the low- $T$ Hall effect that does not follow the expected 'Luttinger' $1+p$ line but instead drops monotonically towards $p$ near OP doping [14] and (iii) a $H$-linear magnetoresistance $(\mathrm{MR})$ at high field strengths 15 that exhibits $H / T$ scaling [16] and is also insensitive to both field orientation and impurity scattering 
rate [16] (for more details, see the introduction to Appendix A). Consequently, this $H$ linear MR has been attributed to incoherent carriers undergoing Planckian dissipation, with a relaxation rate $\hbar / \tau \sim k_{B} T$ [16]. The form of the (magneto)resistivity is similar to that found in other correlated metals close to a quantum critical point (QCP) [17 -19]. Uniquely, in OD cuprates, this $T$ - and $H$-linear component of the resistivity exists across the entire strange metal regime, suggesting strongly that it is not tied to any singular QCP [20]. Similarly, the observed loss of carriers across this regime is difficult to reconcile with the absence of any pseudogap features, e.g. in the electronic specific heat 21] above $p^{*}$. Collectively, these features establish OD cuprates as exceptional non-Fermi-liquids harboring two distinct charge sectors; one associated with coherent quasiparticles, the other seemingly incoherent and non-quasiparticle in nature [16].

Given the presence of these two sectors, it is pertinent to pose the question: which sector is responsible for (high-temperature) superconductivity? Here we show, with a minimal set of assumptions, that with decreasing doping, the superfluid density at zero temperature $\left(n_{s}(0)\right)$ in Tl2201 and LSCO grows at the expense of the coherent carrier density $\left(n_{c o h}\right)$. This correspondence leads us to postulate that superconductivity within the strange metal phase of OD cuprates is not, as expected, an instability of the FL, but rather an instability of the incoherent non-FL sector.

\section{Superfluid and coherent carrier density in $\mathrm{Tl}_{2} \mathrm{Ba}_{2} \mathrm{CuO}_{6+\delta}$}

The key quantities for our analysis are the Hall number $n_{\mathrm{H}}(0)$ and the superfluid density $n_{s}(0)$. For a cuprate like Tl2201 with a barrel-shaped FS [22,23] and an almost isotropic (in-plane) Fermi velocity $v_{F}$ or effective mass $m^{*}$, the former can be expressed simply as

$$
n_{\mathrm{H}}(0)=V_{\text {cell }} /\left(R_{\mathrm{H}}(0) e\right)
$$

where $R_{\mathrm{H}}(0)$ is the as-measured Hall coefficient in the low- $T$, high-field limit (i.e., once any residual anisotropy is washed out $[14 \mid)$ and $V_{\text {cell }}$ is the unit cell volume. Similarly, $n_{s}(0)$ can be derived from the London equation:

$$
n_{s}(0)=\left(m^{*} V_{\text {cell }}\right) /\left(\mu_{0} e^{2} \lambda_{a b}^{2}(0)\right)
$$

where $\lambda_{a b}(0)$ is the in-plane zero-temperature penetration depth. In an ordinary onecomponent Galilean invariant FL, correlation effects do not cause a renormalisation of $\lambda(0)$ and hence, one must use the bare electron mass to estimate $n_{s}(0)$ [24. In heavy fermion systems [25] and iron-pnictides [26], however, $\lambda_{a b}(0)$ is found to be renormalised by the thermodynamic mass $m^{*}$, the breaking of Galilean invariance attributed to the presence of two independent components. It is reasonable to infer that the two sectors considered here might also be responsible for the breaking of translational invariance in OD cuprates, though other factors, including strong correlations [27, disorder and/or Umklapp scattering could also be playing a role.

To motivate the use and indeed choice of the thermodynamic mass in Eq. (2) for OD cuprates, we compare in Figure 1A the fraction of carriers that condense into the superconducting state in OD Tl2201 determined via two independent routes. The closed triangles in Figure $1 \mathrm{~A}$ represent the ratio $n_{s}(0) /(1+p)$, where $n_{s}(0)$ has been extracted from muon-spin relaxation $(\mu \mathrm{SR})[4,5,28]$ and microwave surface impedance [6] measurements of $1 / \lambda_{a b}^{2}(0)$, using Eq. (2) and assuming $m^{*}=5.2 m_{e}$. This doping-independent value for $m^{*}$ matches that obtained from quantum oscillation (QO) experiments on single crystals with $T_{c}=10 \mathrm{~K}$ and $\left.27 \mathrm{~K} 29\right]$ as well as the normal state electronic specific heat 
A

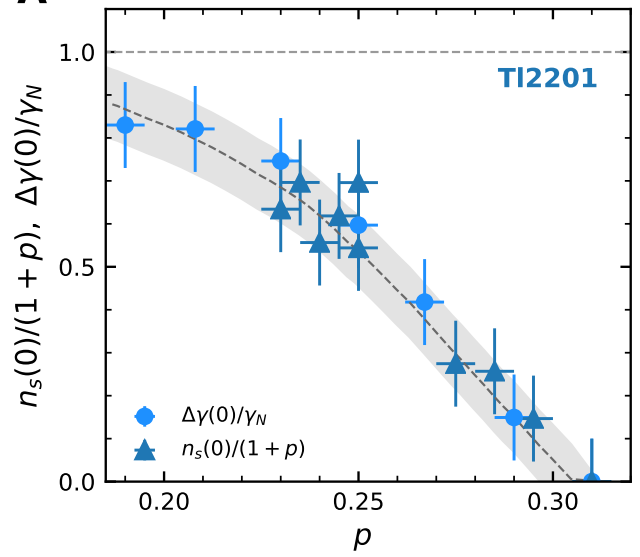

B

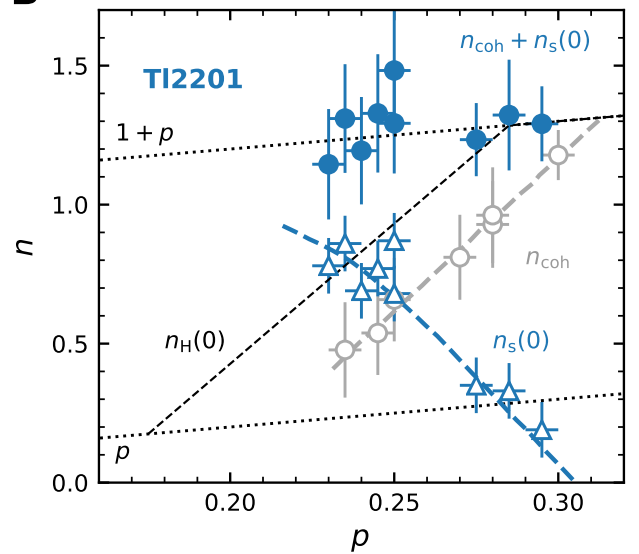

Figure 1: Superfluid density and coherent normal-state carrier density in overdoped Tl2201. A) Comparison of $n_{s}(0) /(1+p)$ and $\Delta \gamma(0) / \gamma_{N}$ in OD Tl2201, where $n_{s}(0) /(1+p)$ (triangles) is the fraction of the total Luttinger count $(1+p)$ that contributes to the as-measured superfluid density $n_{s}(0)[4,6,28]$ and $\Delta \gamma(0) / \gamma_{N}$ (circles) is the fractional change in the electronic specific heat coefficient in the superconducting state $\Delta \gamma(0)$ relative to its value $\gamma_{N}$ in the normal state 30,31$] . n_{s}(0)$ is determined from $1 / \lambda_{a b}^{2}(0)$ using Eq. (2) with $m^{*}=5.2 m_{e}$, consistent with $\gamma_{N}$ for OD Tl2201 across the doping series. The grey shaded region and dashed lines are guides to the eye. B) Open triangles: $n_{s}(0)$ values transposed from Panel A. Black dashed line: schematic $n_{\mathrm{H}}(0)$ line for Tl2201 derived from high-field Hall effect measurements [14]. Open circles: $n_{\text {coh }}$ values for Tl2201, obtained by renormalising the as-measured $n_{\mathrm{H}}(0)$ by the ratio (squared) of the coherent contribution to the total conductivity at $0 \mathrm{~K}$, as determined by analysis of $\rho_{a b}(T)$ (see appendix, section A for details). The faint dashed line is a guide to the eye. Blue filled circles: The sum $n_{c o h}+n_{s}(0)$ for Tl2201. For each value of $n_{s}(0)$, the corresponding value of $n_{c o h}$ was read off from the faint dashed line. The two thin dotted lines represent the relation $n=p$ and $n=1+p$. The estimates of the error bars for each set of data are described in detail in the appendix, section $\mathrm{A}$.

coefficient $\gamma_{N}$ for $0 \mathrm{~K} \leq T_{c} \leq 80 \mathrm{~K}[30]$. The values for $(1+p)$ - the full Luttinger count - are determined from the value of $T_{c}$ as described in Ref. 14]. The circles in Fig. 11A represent $\Delta \gamma(0) / \gamma_{N}$ where $\Delta \gamma(0)=\gamma_{N}-\gamma(0)$ and $\gamma(0)$ is the residual electronic specific heat in the zero-temperature limit [31]. Hence, $\Delta \gamma(0) / \gamma_{N}$ represents the fraction of states that enter into the superconducting condensate. Note that both fractions appear to approach unity around $p=0.19$.

It is important to realise that such excellent agreement between the two quantities shown in Fig. 1 $\mathrm{A}$ is not a trivial finding. While $\Delta \gamma(0) / \gamma_{N}$ is independent of $m^{*}$, the conversion from $1 / \lambda_{a b}^{2}(0)$ to $n_{s}(0)$ in Eq. (2) relies solely on the magnitude of $m^{*}$, which is itself determined independently by measurements of $\gamma_{N}$ (as well as QO). Rather, this robust consistency between the two quantities - ostensibly across the entire OD regime of Tl2201 - confirms the need to invoke the thermodynamic mass in the evaluation of $n_{s}(0)$ in OD cuprates. For Tl2201 specifically, it confirms both the doping independence of $m^{*}$, even as the doping level $p^{*}=0.19$ associated with the opening of the normal state pseudogap is approached, and the lack of in-plane anisotropy in $m^{*}$ or in $v_{F}$ throughout the doping series (otherwise Eq. (2) would not be valid). This lack of anisotropy is consistent with the expectation that the Fermi level in superconducting Tl2201 lies well above the van Hove singularity (vHs) 14,32 . 
Having established the doping dependence of $n_{s}(0)$, we next turn to examine the evolution of the coherent carrier density $n_{c o h}$. The main result is summarized in Fig. $11 \mathrm{~B}$. The open triangles in Fig. $1 \mathrm{~B}$ are the same $n_{s}(0)$ values transposed from Panel A. The black dashed line represents schematically the evolution of $n_{\mathrm{H}}(0)$ across the strange metal regime in OD Tl2201 derived from high-field Hall effect measurements [14. The anticorrelation between $n_{s}(0)$ and $n_{\mathrm{H}}(0)$ in Fig. $1 \mathrm{~B}$ is clear - as the Hall number increases toward $1+p$, the superfluid density falls, at a similar rate, toward zero. While this finding is already striking and anomalous, it is $n_{c o h}$ that is most relevant here, not $n_{\mathrm{H}}(0)$. Recall that $n_{\mathrm{H}}(0)$ is obtained directly from the measured Hall voltage via Eq. (1) and is not necessarily equivalent to $n_{c o h}$, even in a system like Tl2201 with a simple, cylindrical FS and an isotropic $v_{F}$. The modifying factor in this case is the presence of the second, incoherent sector deduced from the in-plane MR studies [16. The precise way in which this second sector modifies the analysis of $R_{\mathrm{H}}(0)$ depends on the nature of the sector and how this manifests itself in the transport properties. Here, we assume that both the coherent and incoherent sectors co-exist on the same FS but are located at different regions in $k$-space, the former near the zone diagonals, the latter along the flat sections of FS near $( \pm \pi, 0)$ and $(0, \pm \pi)$. In this way, their contributions to the total conductivity are additive, as they would be in a normal two-fluid system. Secondly, we assume that the Hall conductivity $\sigma_{x y}$ of the incoherent sector is zero, as inferred in Ref. [14]. Based on these simplifying assumptions, $n_{c o h}$ is found to be related to $n_{\mathrm{H}}(0)$ via the following simple expression:

$$
n_{c o h}=f_{\sigma} n_{\mathrm{H}}(0)
$$

where $f_{\sigma}=\left(\sigma_{x x}^{c o h} / \sigma_{x x}^{t o t}\right)^{2}$ is the square of the contribution of the coherent sector $\sigma_{x x}^{c o h}$ to the total dc conductivity $\sigma_{x x}^{\text {tot }}$ (see appendix, section A.1 for full details). Since $f_{\sigma} \leq 1$, $n_{\text {coh }} \leq n_{\mathrm{H}}(0)$ for all dopings. Estimates of $f_{\sigma}$ for Tl2201 at various $p$ values, deduced from fitting the zero-field resistivity, are presented in appendix $\mathrm{A}$. The resultant $n_{c o h}$ values are plotted as open circles in Fig. 1B. Note that $n_{\text {coh }}$ appears to reach the $1+p$ line at $p \sim$ 0.31 , i.e. where both superconductivity and the $T$-linear term in $\rho_{a b}(T)$ vanish [13].

In an ordinary BCS superconductor (in the clean limit), $n_{s}(0)=n_{\text {coh }}$ as all (mobile) electrons condense into the superfluid. In OD cuprates, however, this appears not to be the case. While disorder might reduce $n_{s}(0)$ through pair-breaking, the concomitant growth in $n_{\text {coh }}$ with doping is wholly unexpected. More revealing, however, is the observation that the sum $n_{c o h}+n_{s}(0) \approx 1+p$ (filled circles in Fig. 1 $1 \mathrm{~B}$ ). This simple quantitative relation is our central finding. Before discussing its implications, however, we first turn to consider whether a similar relation may also hold in another OD cuprate, namely $\mathrm{La}_{2-x} \mathrm{Sr}_{x} \mathrm{CuO}_{4}$.

\section{Superfluid and coherent carrier density in $\mathrm{La}_{2-x} \mathrm{Sr}_{x} \mathrm{CuO}_{4}$}

While the doping range of several cuprate families extends beyond $p^{*}$, only LSCO has been studied in sufficient detail to enable us to investigate how $n_{c o h}$ and $n_{s}(0)$ evolve with doping across the entire strange metal regime, i.e. between $p^{*}$ and $p_{S C}-$ the doping level corresponding to the edge of the superconducting dome. Extracting both quantities in OD LSCO, however, is not as straightforward as it is in Tl2201, due to the distinct FS topology and strong in-plane anisotropies of the former. While in Tl2201, the FS remains hole-like far beyond $p_{S C}$ [14], the FS in LSCO transitions from hole-like to electron-like around $p=0.195$ due to the Fermi level crossing the vHs at $(\pi, 0)$ 33. As a result, the FS of OD LSCO contains not only significant anisotropy in $v_{F}$ (due to proximity of the Fermi level to the saddle point at $(\pi, 0)$ ), but also regions of electron- and hole-like 
curvature (see Fig. 2A for an illustration, note that the labels inc and coh do not necessarily correspond explicitly to the curvature). In addition, its impurity scattering rate $1 / \tau_{0}$ (i.e., in the zero-temperature limit) has also been shown to be highly anisotropic [34, 35 due to a predominance of small-angle scattering off impurities located outside of the $\mathrm{CuO}_{2}$ planes 36. The anisotropies in $v_{F}$ and $1 / \tau_{0}$ thus conspire, rather than cancel, to produce a zero-temperature mean-free-path $\ell_{0}$ that can be up to two orders of magnitude larger at the zone edge than along the zone diagonals [35]. This extreme anisotropy, coupled with the change in curvature around the (in-plane) FS, means that the relation between $R_{\mathrm{H}}(0)$ and $n_{\mathrm{H}}(0)$ specified in Eq. (1) is no longer meaningful. Hence, even within a picture based solely on coherent quasiparticles, there are several aspects that need to be taken into account before the possible influence of any incoherent states can be considered.

With this in mind, we set out to model self-consistently the as-measured transport and thermodynamic quantities of OD LSCO via the following strategy. We take as our starting point the two-dimensional (2D) tight-binding FS parameterization at various doping levels, as determined by angle-resolved photoemission spectroscopy (ARPES) 33. We assume that any uncertainty introduced by ignoring the dispersion along $k_{z}$ is negligible, its effect being averaged out in any full 3D integral. We then use this $2 \mathrm{D}$ parameterization, along with the corresponding as-measured $v_{F}(\phi)$, to calculate an estimate for the normal state electronic specific heat coefficient $\gamma_{N}$ using the expression:

$$
\gamma_{N}=\frac{V_{\text {cell }} k_{B}^{2} N_{A}}{12 \pi \hbar} \oint d S \frac{k_{F}(\phi)}{\left|v_{F}(\phi)\right| \cos \delta}
$$

Here, the units of $\gamma_{N}$ are $\mathrm{J} / \mathrm{mol} \cdot \mathrm{K}^{2}, \phi$ is the in-plane azimuthal angle, with $\phi=0$ corresponding to the $k$-vector $(\pi, 0)$, and $\delta$ is the angle between $\mathbf{v}_{\mathbf{F}}$ and the Fermi wave vector $\mathbf{k}_{\mathbf{F}}$ (that ensures that the correct gradient is used for the calculation of $\gamma_{N}$ ). Taking $v_{F}(\phi)$ directly from ARPES, however, generates an estimate for $\gamma_{N}$ that is approximately one half of the experimentally-determined value [33]. The origin of this discrepancy is not yet known; it may reflect an additional low-energy renormalization in the quasiparticle dispersion - not detected by ARPES in LSCO, but seen, for example, in Bi2201 below $2-4 \mathrm{meV}$ [41] - or something more fundamental. In order to proceed, we simply scale the absolute value of $\left|v_{F}(\phi)\right|$ to match $\gamma_{N}$ for each representative doping level while maintaining its ARPES-derived $\phi$ dependence (since this appears to have been confirmed by different magneto-transport measurements [34,35]). The resultant scaling values are shown in Table 8 in Appendix B. In this way, $\gamma_{N}(x)$ is taken to be the physical quantity against which all other quantities are determined. This choice is motivated by (i) the fact that measurements of $\gamma_{N}(x)$ in OD LSCO by different groups are in good agreement and show a systematic evolution with $x$ [38, 42 44], and (ii) the expectation that both the coherent and incoherent sectors will contribute to the total density of states.

Thus, by combining specific heat and ARPES data, we are enable to define, with reasonable confidence, $k_{F}(\phi)$ and $v_{F}(\phi)$ across the entire doping range of interest, i.e. 0.20 $\leq x, p \leq 0.32$. The next step is then to compare the as-measured values of $1 / \lambda_{a b}^{2}(0), 7,37$ with those computed from the full integral formula:

$$
\frac{1}{\lambda_{a b}^{2}(0)}=\frac{\mu_{0} e^{2}}{4 \pi^{3} \hbar} \oint d S \frac{v_{x}^{2}}{\left|v_{F}\right| \cos \delta}
$$

Here, $v_{x}$ is the $x$-component of $v_{F}$. The resulting comparisons are shown in Table 9 in Appendix B. Clearly, as in OD Tl2201, only a fraction of the total states contribute to $n_{s}(0)$. At this stage, we make no assumption about which sector generates the superfluid in LSCO, but instead consider two scenarios $C(I)$ - in which the superfluid emerges purely from coherent (incoherent) states, respectively - on an equal footing. Taking our cue from 
A
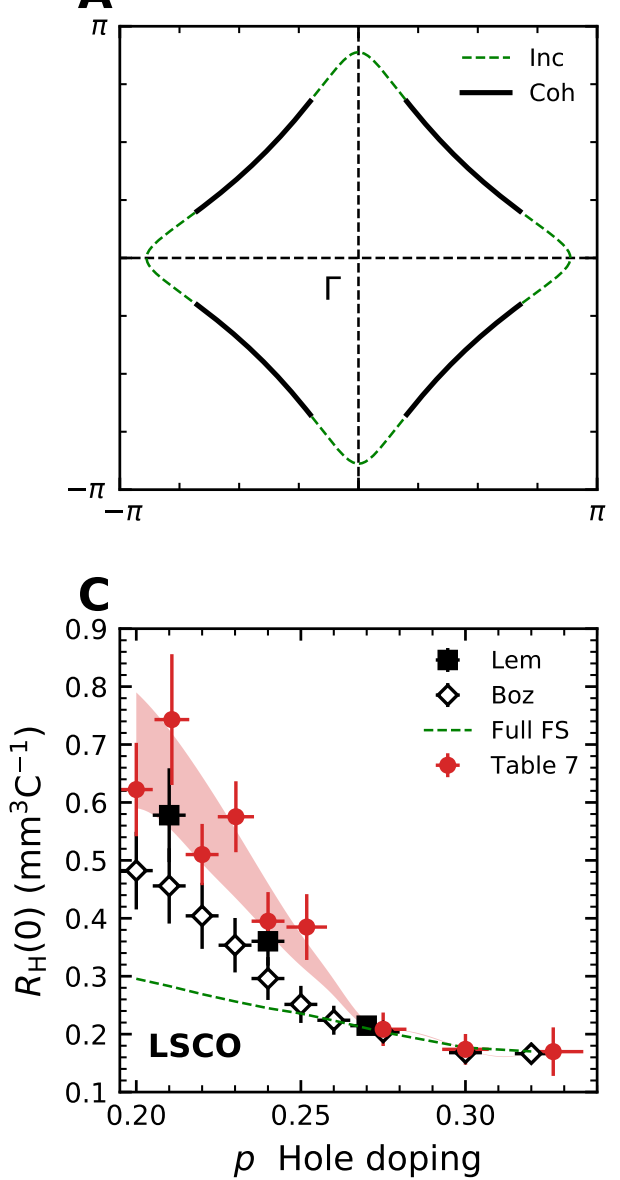

B

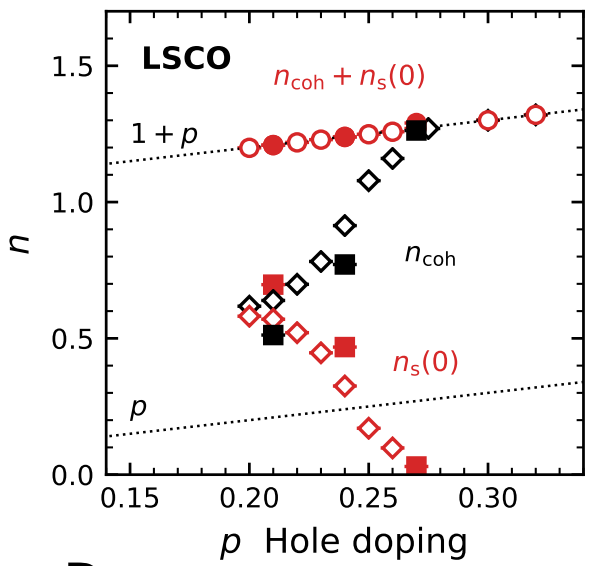

D

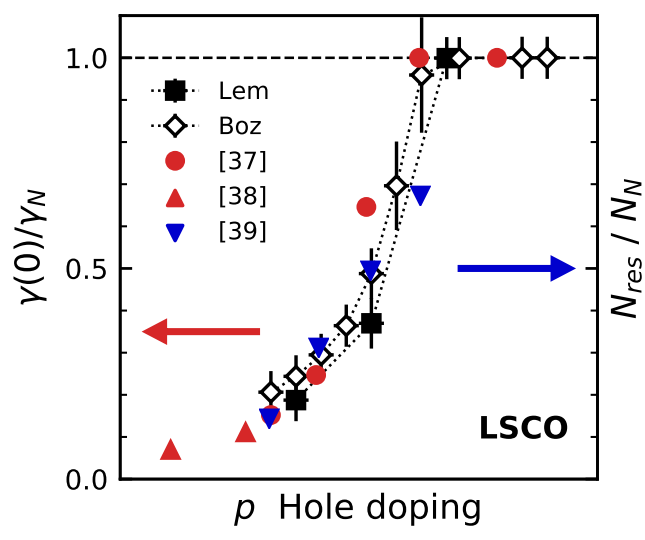

Figure 2: Possible break-up of the Fermi surface in OD LSCO into coherent and incoherent sections and its possible consequences. A) Example of a truncated FS (corresponding to $p=x=0.23$ ) in which anti-nodal sections (green dashed lines) exhibit incoherent charge dynamics and the nodal sections (bold black lines) are coherent. Note that the full FS is seen in ARPES. B) $n_{s}(0)$ at selected dopings extracted from mutual inductance measurements of $1 / \lambda_{a b}^{2}(0)$ in LSCO thin films by Božović et al. (open red diamonds) [7] and Lemberger et al. (filled red squares) [37]. Black empty diamonds (filled squares) represent $n_{\text {coh }}$, the density of coherent carriers at low- $T$ deduced from each corresponding data set, assuming $n_{c o h}=(1+p)-n_{s}(0)$. C) Doping dependence of $R_{\mathrm{H}}(0)$ in OD LSCO. (Red circles) Binned and averaged literature values for $R_{\mathrm{H}}(0)$ (see Table 7). Red shaded area indicates the spread in the (binned and averaged) experimental values. Green dashed line represents the doping dependence of $R_{\mathrm{H}}(0)$ calculated using Boltzmann transport theory and assuming the entire FS is coherent. Note the clear bifurcation between experimental and Boltzmann-derived values of $R_{\mathrm{H}}(0)$ at $p_{S C}=0.27$. Open diamonds (filled squares) represent the calculated $R_{\mathrm{H}}(0)$ obtained by truncating the FS integral for $\sigma_{x y}$ as constrained by the $n_{c o h}$ values shown in panel $\mathbf{B}$ and deduced from the Božović [7] (Lemberger [37]) $n_{s}(0)$ values. D) Residual density of states within the SC state of OD LSCO as determined by specific heat (circles [38 and triangles 39]) and Knight shift (inverted triangles) [40] studies. Again, open diamonds (filled squares) represent the residual density of states calculated using the same truncated FS integrals employed in panel $\mathbf{C}$ and the $n_{s}(0)$ values deduced from Ref. [7] ( [37]), respectively. The error analysis for all panels is described in Appendix $B$. 
the magneto-transport measurements 34, 35, however, we assume, as in Tl2201, that the coherent states are located near $(\pi, \pi)$ while the incoherent states reside near $(\pi, 0)$. We then proceed by truncating the bounds of the integral in Eq. (5) at each $x$ until the calculated $1 / \lambda_{a b}^{2}(0)$ matches the experimental value (see Fig. 2A for an example of such truncation). The bounds themselves lead to a direct estimate of $n_{s}(0)$. Once $1 / \lambda_{a b}^{2}(0)$ is matched, we then calculate $\gamma(0)$ and $R_{\mathrm{H}}(0)$ - using precisely the same bounds - and compare these with their corresponding experimental values (where known). The full set of results for scenario $I$ are summarized in Fig. 2 (see appendix B for details). Scenario $C$ is discussed in appendix $B$.

Before comparing the outcomes from both scenarios, let us first elaborate on how $R_{\mathrm{H}}(0)$ is calculated and then compared with experiment. As mentioned above, the variable FS curvature in OD LSCO gives contributions to $\sigma_{x y}$ of opposite sign 45]. This feature, combined with the violation of the isotropic- $\ell$ approximation, means that the simple Drude relation between $R_{\mathrm{H}}(0)$ and $n_{\mathrm{H}}(0)$ breaks down and the full Boltzmann expressions for both $\sigma_{x x}$ and $\sigma_{x y}[46]$ need to be considered. (The expressions themselves are described in Appendix B.3.) Reassuringly, as shown in Fig. 2 $\mathrm{B}$, an excellent quantitative match to the experimental $R_{\mathrm{H}}(0)$ values is found for dopings $p>p_{S C}$ using the full integrals. This agreement implies that the FS for non-SC OD LSCO is fully coherent, consistent with reports that the ground state of this region in the phase diagram is that of a correlated FL 44. Below $p_{S C}$, however, the as-measured and FS-derived values for $R_{\mathrm{H}}(0)$ appear to deviate from each other (the shaded region indicates the spread in $R_{\mathrm{H}}(0)$ values from the literature - see Appendix B.3 for details). In this regime, the experimental $R_{\mathrm{H}}(0)$ values can only be reconciled with the full integral formulae for $\sigma_{x x}$ and $\sigma_{x y}$ either by increasing $\delta$ (i.e. the effective FS curvature) or by increasing the anisotropy in $v_{F}(\phi)$ to values that are extreme and vastly different to those determined by ARPES [33 (again see Appendix B.3 for details). The more self-consistent way to account for this change of slope in $R_{\mathrm{H}}(0)(x)$, however, is to invoke the presence of the two sectors below $p_{S C}$, to truncate the bounds of the FS integrals for both $\sigma_{x x}$ and $\sigma_{x y}$ (as done for $1 / \lambda_{a b}^{2}(0)$ and $\gamma(0)$ ) and to set $\sigma_{x y}=0$ in the incoherent sector (as done for OD Tl2201). Within scenario $I$, the region near $(\pi$, 0 ) is the one which gives the positive (negative) contribution to $\sigma_{x y}\left(R_{\mathrm{H}}(0)\right)$ [45. Thus, by again setting $\sigma_{x y}=0$ in this region, the net effect is to make the calculated value of $R_{\mathrm{H}}(0)$ more positive, thereby leading to a better match to the experimental value.

The black squares (diamonds) in Fig. $2 \mathrm{C}$ represent $R_{\mathrm{H}}(0)$ values for OD LSCO obtained using the same FS parameterization and integral bounds that were used to match the $1 / \lambda_{a b}^{2}(0)$ values at each respective $x$ (within scenario $I$ ). Good agreement between the calculated and experimental values is now found for all $x$. Note that in all cases, we have assumed $f_{\sigma}=1$. This simplifying assumption is reasonable in LSCO given the huge anisotropy in $\ell_{0}$ discussed above (see Appendix B.4 for more detail). The corresponding comparison for $\gamma(0) / \gamma_{N}$ is shown in Fig. 2D. Again, for scenario $I$, the overall trend is reproduced across the entire strange metal regime of OD LSCO. Hence, despite the very marked difference in maximum $T_{c}$, FS topology and disorder level in LSCO compared to Tl2201, the collective Hall density, superfluid density and specific heat data appear consistent with the same relation, i.e. $n_{c o h}+n_{s}(0)=1+p$.

As discussed in Appendix B, the alternative model (scenario $C$ ), with the condensate derived from near-nodal states, fails to give a consistent match between $n_{s}(0)$ and $\gamma(0) / \gamma_{N}$. Of course, pair breaking in a $d$-wave superconductor is expected to affect predominantly the 'nodal' regions where the gap is smallest. Thus, the conjecture presented here is not necessarily a unique solution. Nevertheless, there are a number of other findings discussed in Appendix B that appear to be in conflict with current predictions from dirty $d$-wave theory applied to OD LSCO. Moreover, the marked $x$-dependence of $R_{\mathrm{H}}(0)$ in OD LSCO 
discussed above can only be modelled self-consistently within scenario $I$. The dirty $d$-wave scenario, by contrast, relies on the existence of a full coherent FS for all $x$.

\section{Discussion}

The anti-correlation between $n_{s}(0)$ and $n_{c o h}$ obtained within scenario $I$ implies that once superconductivity is suppressed, either by magnetic field or by temperature, the condensed carriers do not re-emerge as coherent quasiparticles. It is as though the superfluid condensate emerges at the expense of the coherent sector. The total carrier density is of course fixed by the Luttinger count. The relation $n_{c o h}+n_{s}(0)=1+p$ then becomes even more constraining, since it implies that the superconducting condensate in OD LSCO and T12201 derives only from those carriers that exhibit signatures of incoherent transport. It is important to remark that while all the key quantities: $n_{s}(0), n_{\mathrm{H}}(0), \gamma(0)$ and $f_{\sigma}$, are defined in the zero temperature limit (either in zero magnetic field or in fields at which the superconductivity is suppressed), $f_{\sigma}$ (in Tl2201) has been deduced from finitetemperature resistivity curves (in LSCO, we have simply assumed that $f_{\sigma} \sim 1$ ). It is the (extrapolated) ratio of the zero-temperature (residual) resistivities of the two sectors that effectively renormalizes $n_{\mathrm{H}}(0)$ to obtain $n_{c o h}$. Nevertheless, we do not expect the ratio of conductivities to vary significantly between $0 \mathrm{~K}$ and $T_{c}$ to affect these extrapolations.

The notion that superconducting coherence emerges out of an incoherent normal state in cuprates is not new [47]. Early ARPES studies showed that a coherent quasiparticle peak evolves below $T_{c}$ at the zone boundary (the so-called anti-nodal region) from an incoherent, normal-state background in UD and OP cuprates, and in addition, the bulk of the superconducting condensate originates from states near $(\pi, 0)[48,49$. A marked enhancement in the microwave [50] or thermal conductivity below $T_{c} \quad 51$ also indicated a dramatic increase in the mean-free-path of uncondensed carriers, while in-plane optical studies of UD or OP $\mathrm{Bi}_{2} \mathrm{Sr}_{2} \mathrm{CaCu}_{2} \mathrm{O}_{8+\delta}$ (Bi2212) revealed that a significant fraction of the superfluid spectral weight comes from energies far above the superconducting gap scale $(4 \Delta)$ 52, 53]. All such features, however, were reported for UD or OP cuprates, and it has been largely assumed that such exotic features become weaker, or even vanish, on the OD side. Spectral weight transfer, for example, was found to be reversed in OD Bi2212, suggesting a possible recovery of conventional BCS condensation [54 56]. It was subsequently noted, however, that the vHs in Bi2212 (on one of the Fermi sheets) may markedly affect the proportionality between spectral weight transfer and the change in kinetic energy across $T_{c}$, potentially masking any intrinsic kinetic-energy saving [57].

How superconductivity evolves as the pseudogap opens below $p^{*}$ lies outside the scope of this work. Nevertheless, we can make some preliminary remarks here. It is well known that $n_{s}(0)$ in hole-doped cuprates peaks at $p^{*}$ and once the pseudogap opens, it drops precipitously [58. At the same time, the signature of incoherent carriers in the normal state MR is lost and conventional [59] or modified [60] Kohler's scaling is recovered. These properties suggest that incoherent carriers are predominantly gapped out below $p^{*}$ and as such are unable to contribute to the superfluid density. It has been largely assumed until now that the remaining states (i.e. on the Fermi arcs) also contribute to $n_{s}(0)$ in UD and OP cuprates. The presence of a residual electronic density of states (finite specific heat at $T=0$ ) in even the very highest quality crystals [39, 61] and the emergence of quantum oscillations at magnetic field strengths below the solid-to-liquid vortex transition [62, may indicate otherwise.

Given the possible implications of our findings for the understanding of high- $T_{c}$ superconductivity, we conclude by highlighting some of its possible caveats. The scenario based 
on independent (parallel) conduction channels presented here assumes that the low-lying states associated with each sector are located in different regions in momentum space (in UD cuprates, this distinction is often referred to as the 'nodal-anti-nodal dichotomy'). While this is easy to conceptualize, it is not a unique interpretation. Moreover, it is not possible at this stage to determine whether a scenario in which the resistivities or the conductivities of the two sectors are added captures the experimental data better, even though the neatness of the obtained relation between $n_{c o h}$ and $n_{s}(0)$ suggests that the parallel scenario is at least a viable starting point.

Secondly, the presence of incoherent states at the Fermi level seems, at first hand, incompatible with the observation of $\mathrm{QO}$ in OD Tl2201 [8]. It is important to note, however, that despite extensive efforts, QOs have only been seen to date at very high dopings levels in Tl2201, i.e. beyond $p=0.27$ (but still within the strange metal regime) [29]. At these doping levels, where the incoherent sector is envisaged to be small (and therefore possibly restricted in $k$-space), quasiparticles may experience the incoherent sector simply as an additional dephasing or modified Dingle term. Indeed, the meanfree-path estimated from the Dingle analysis of QOs in heavily OD Tl2201 is roughly a factor of 2 shorter than that obtained from the in-plane resistivity (until now this discrepancy has been attributed to different contributions from small- and large-angle scattering). Alternatively, coherent quasiparticles may be able to traverse sufficiently narrow incoherent sections similar to how magnetic breakdown in a system with multiple pockets enables quasiparticles to tunnel across the breakdown gap. Both scenarios provide a mechanism by which QO can still be observed, provided the magnetic field is strong enough, though clearly QO studies on Tl2201 crystals doped across $p_{S C}$ would help to identify if there is indeed any additional dephasing present.

Thirdly, one curious outcome of this analysis is the extended range of coexistence of the coherent and incoherent channels in OD Tl2201 relative to LSCO that echoes the extended range of superconductivity in the former. Within a dirty $d$-wave scenario for the cuprates, the reason for the extended range of superconductivity in Tl2201 on the OD side is obvious; lower disorder levels simply induce less pair-breaking. Indeed, it has been argued that both the variation of $n_{s}(0)$ with $T_{c}$ in LSCO (and Tl2201) and the $T$ dependence of $n_{s}(T)$ can be captured well by the dirty $d$-wave picture [63 65]. As shown in Appendix C, however, the magnitude of the normal state scattering rate (obtained from the residual resistivity) in OD cuprates can be, when converted into units of temperature, more than one order of magnitude larger than the corresponding $T_{c}$ values. Such large values for the normal state scattering rate should, according to the predictions, extinguish superconductivity entirely, irrespective of whether the scattering itself is in the Born or unitary limit. Moreover, variations in the residual resistivity appear to have little or no influence on $T_{c}$ itself, in marked contrast with expectations from the theory. In light of the analysis presented here, a re-examination of other claims of compatibility with the dirty $d$-wave scenario for OD cuprates may be timely.

Finally, we note that a recent phenomenological model by Pelc et al. has also invoked the co-existence of two sub-systems - one itinerant and FL-like, the other localized across the OD superconducting regime of hole-doped cuprates; their sum recovering the full Luttinger count. As postulated here, the superfluid density in that picture is argued to derive from the localized, rather than itinerant carriers 66. According to their model, the density of mobile carriers decreases continuously as $T$ falls below the effective (inhomogeneous) localization gap while their scattering rate maintains a strict $T^{2}$ dependence. Although similar in spirit to our own proposal, this picture is inconsistent with the observed drop in $R_{\mathrm{H}}(T)$ at low $T$ and the quadrature scaling of the MR 16 which imposes a component in the zero-field resistivity with a $T$-linear (Planckian) scattering rate. 


\section{Conclusion}

Taking as our motivation recent high-field magnetotransport measurements showing signatures of coherent and incoherent transport in the strange metal phase of overdoped cuprates, we have re-examined the London penetration depth, specific heat and Hall effect in overdoped $\mathrm{Tl}_{2} \mathrm{Ba}_{2} \mathrm{CuO}_{6+\delta}$ and $\mathrm{La}_{2-x} \mathrm{Sr}_{x} \mathrm{CuO}_{4}$. Our analysis reveals that the existing experimental data can be reconciled with a scenario in which these coherent and incoherent carriers are located on distinct regions of the underlying Fermi surface. Based on the assumption that the conductivities of these two sectors add in parallel, we have shown that in $\mathrm{Tl}_{2} \mathrm{Ba}_{2} \mathrm{CuO}_{6+\delta}$, the growth of the superfluid density $n_{s}(0)$ with decreasing doping $p$ is quantitatively compensated with the decrease in the coherent carrier density $n_{c o h}$ and that their sum is approximately equal to the full Luttinger count $1+p$. Assuming a similar relation for $\mathrm{La}_{2-x} \mathrm{Sr}_{x} \mathrm{CuO}_{4}$, we find good consistency in the evolution of the limiting low$T$ Hall coefficient as well as the residual specific heat (inside the superconducting state). These correspondences, if confirmed, could indicate that, in contrast to the standard BCS theory, superconductivity in both $\mathrm{Tl}_{2} \mathrm{Ba}_{2} \mathrm{CuO}_{6+\delta}$ and $\mathrm{La}_{2-x} \mathrm{Sr}_{x} \mathrm{CuO}_{4}$ emerges from states that exhibit incoherent, rather than coherent transport in the normal state. Finally, the anti-correlation between $n_{s}(0)$ and $n_{c o h}$ coupled with the non-FL transport properties exhibited right across the overdoped region, challenges previous notions that dirty $d$-wave BCS picture is an appropriate starting point for a description of OD cuprates. Evidently, OD cuprates need the strange metal phase in order to become superconducting.

\section{Acknowledgements}

The authors would like to acknowledge stimulating discussions with M. Allan, I. Božović, M. S. Golden, B. Goutéraux, C. Pépin, K. S. Schalm, E. van Heumen and J. Zaanen.

Funding information We acknowledge the support of the HFML-RU/NWO, a member of the European Magnetic Field Laboratory (EMFL). This work is part of the research programme 'Strange Metals' (grant number 16METL01) of the former Foundation for Fundamental Research on Matter (FOM), which is financially supported by the Netherlands Organisation for Scientific Research (NWO). This work was also supported by the European Research Council (ERC) under the European Union's Horizon 2020 research and innovation programme (Grant Agreement No. 835279-Catch-22).

\section{A Obtaining estimates for $n_{\text {coh }}$ and $n_{s}(0)$ in $\mathbf{T l}_{2} \mathrm{Ba}_{2} \mathrm{CuO}_{6+\delta}$}

In a recent study, the low- $T$ Hall coefficient $R_{\mathrm{H}}(0)$ of overdoped (OD) $\mathrm{Tl}_{2} \mathrm{Ba}_{2} \mathrm{CuO}_{6+\delta}$ (Tl2201) was measured for various doping levels in magnetic fields large enough to access the non-superconducting ground state [14. At the lowest temperatures, the Hall resistivity $\rho_{x y}(H)$ was found to be linear in magnetic field strength $H$ and as a consequence, $R_{\mathrm{H}}(0)$ was obtained from the asymptotic low- $T$ high- $H$ limit of $\rho_{x y} / H$, from which the low- $T$ Hall number $n_{\mathrm{H}}(0)=V_{\text {cell }} /\left(R_{\mathrm{H}}(0) e\right)$ was then determined. The evolution of $n_{\mathrm{H}}(0)$ with doping is shown schematically as a faint dashed line in Fig. 1B.

In that work, it was assumed that $n_{\mathrm{H}}(0)$ represented the number density of coherent charge carriers in OD Tl2201. A subsequent study of the in-plane magnetoresistance (MR), however, revealed evidence for incoherent carriers within the $\mathrm{CuO}_{2}$ planes in addition to the coherent quasiparticles [16]. Specifically, the magnitude of the MR was found to be 
at least one order of magnitude larger than expected, given the residual resistivity and corresponding (impurity) scattering rate. Its magnitude was also found to be insensitive to the orientation of the applied field, suggesting that the observed MR was non-orbital in nature. Moreover, the precise $H / T$ scaling seen in the MR implied that its origin was tied to that of the zero-field $T$-linear resistivity and associated with Planckian dissipation of the relevant carriers.

Collectively, these observations provide compelling evidence for the existence of two conducting sectors within the strange metal regime of OD Tl2201; one coherent, the other incoherent. Until now, however, it has not been possible to determine whether the conductivities of each sector add in parallel (as they would, for example, if they originated from different regions in $k$-space) or in series (e.g. if they originated from different scattering mechanisms). In this report, we consider the former and below, we show how within such a picture, the presence of the second (incoherent) sector modifies the determination of $n_{c o h}$, the number density of coherent carriers, that contribute to the as-measured $\rho_{x y}(H)$ and $R_{\mathrm{H}}(0)$.

\section{A.1 Additive conductivity channels in $\mathrm{Tl}_{2} \mathrm{Ba}_{2} \mathrm{CuO}_{6+\delta}$}

Within a parallel conductivity scenario, we can write the total conductivity $\sigma_{x x}^{\text {tot }}$ as

$$
\sigma_{x x}^{t o t}=\sigma_{x x}^{c o h}+\sigma_{x x}^{i n c}
$$

where $\sigma_{x x}^{c o h}$ and $\sigma_{x x}^{i n c}$ are the coherent and incoherent components respectively. Similarly, the total Hall conductivity $\sigma_{x y}^{\text {tot }}$ can be decomposed into coherent $\left(\sigma_{x y}^{c o h}\right)$ and incoherent $\left(\sigma_{x y}^{i n c}\right)$ contributions, i.e.

$$
\sigma_{x y}^{t o t}=\sigma_{x y}^{c o h}+\sigma_{x y}^{i n c}
$$

After standard matrix inversion, the total Hall resistivity $\rho_{x y}^{\text {tot }}$ becomes a weighted sum of the coherent $\left(\rho_{x x}^{c o h}, \rho_{x y}^{c o h}\right)$ and incoherent $\left(\rho_{x x}^{i n c}\right.$ and $\left.\rho_{x y}^{i n c}\right)$ longitudinal and Hall resistivities respectively

$$
\rho_{x y}^{t o t}=\rho_{x y}^{c o h} \frac{\left(\rho_{x x}^{t o t}\right)^{2}+\left(\rho_{x y}^{t o t}\right)^{2}}{\left(\rho_{x x}^{c o h}\right)^{2}+\left(\rho_{x y}^{c o h}\right)^{2}}+\rho_{x y}^{i n c} \frac{\left(\rho_{x x}^{t o t}\right)^{2}+\left(\rho_{x y}^{t o t}\right)^{2}}{\left(\rho_{x x}^{i n c}\right)^{2}+\left(\rho_{x y}^{i n c}\right)^{2}}
$$

As mentioned above, the incoherent sector in OD Tl2201 exhibits an in-plane MR of a non-orbital origin [16. Similarly, the evolution of $R_{\mathrm{H}}(0)$ in OD Tl2201 indicates that the Hall response from the incoherent sector is negligible [14, as indeed one might expect if orbital motion is impeded. With this in mind, we assume here that the contribution to the Hall effect from the incoherent sector is negligible, i.e.

$$
\rho_{x y}^{i n c}=0
$$

and taking the approximation

$$
\begin{gathered}
\left(\rho_{x x}^{t o t}\right)^{2} \gg\left(\rho_{x y}^{t o t}\right)^{2} \\
\left(\rho_{x x}^{c o h}\right)^{2} \gg\left(\rho_{x y}^{c o h}\right)^{2}
\end{gathered}
$$

as well as assuming a negligible change of the diagonal resistivity components in a magnetic field

$$
\begin{gathered}
\rho_{x x}^{t o t} \approx \rho_{x x}^{t o t}(H=0)=\rho_{x x, 0}^{t o t} \\
\rho_{x x}^{c o h} \approx \rho_{x x}^{c o h}(H=0)=\rho_{x x, 0}^{c o h}
\end{gathered}
$$


Eq. (8) can be simplified to

$$
\rho_{x y}^{t o t} \approx \rho_{x y}^{c o h} \frac{\left(\sigma_{x x, 0}^{c o h}\right)^{2}}{\left(\sigma_{x x, 0}^{t o t}\right)^{2}}
$$

(The validity of approximations (10) and (11) will be discussed further in section A.5. Thus, even though the incoherent sector does not contribute to the Hall conductivity, the as-measured Hall resistivity must be rescaled by the weighting factor

$$
f_{\sigma}=\frac{\left(\sigma_{x x, 0}^{c o h}\right)^{2}}{\left(\sigma_{x x, 0}^{t o t}\right)^{2}}
$$

in order to obtain $\rho_{x y}^{c o h}$, the intrinsic Hall resistivity of the coherent sector. As will become clear, the weighting factor $f_{\sigma}$ plays a key role in determining $n_{c o h}$.

The total $\left(\rho_{x y}^{t o t}(0)\right)$ and coherent $\left(\rho_{x y}^{c o h}(0)\right)$ zero-temperature Hall resistivities can be expressed as

$$
\begin{array}{r}
\rho_{x y}^{\text {tot }}(0)=R_{\mathrm{H}}(0) \mu_{0} H \\
\rho_{x y}^{c o h}(0)=R_{\mathrm{H}}^{c o h}(0) \mu_{0} H
\end{array}
$$

Note that we have removed the suffix "tot" from $R_{\mathrm{H}}^{\text {tot }}(0)$ to be consistent with the labelling in the main text and to emphasize that $R_{\mathrm{H}}(0)$ is the as-measured Hall coefficient in the zero-temperature limit. In standard Drude notation, these Hall coefficients can be expressed as

$$
\begin{aligned}
& R_{\mathrm{H}}(0)=\frac{V_{\text {cell }}}{n_{\mathrm{H}}(0) e} \\
& R_{\mathrm{H}}^{\text {coh }}(0)=\frac{V_{\text {cell }}}{n_{\text {coh } e}}
\end{aligned}
$$

Thus, $n_{\mathrm{H}}(0)$ is directly obtained from measurements of $\rho_{x y}^{\text {tot }}(0)$. However, because the incoherent sector affects $\sigma_{x x}^{t o t}$ without contributing to $\sigma_{x y}^{t o t}, \rho_{x y}^{t o t}(0)$ does not represent the true coherent carrier density, even at high field and low temperature. Similarly, $n_{\mathrm{H}}(0)$ is an experimentally-derived quantity that does not reflect the actual coherent carrier density $n_{\text {coh }}$. The relation between the two is obtained by combining Eqs. 12)-(15)

$$
n_{c o h}=f_{\sigma} n_{\mathrm{H}}(0)
$$

This is the same expression as that given in Eq. (3). Here, $n_{\text {coh }}$ represents the contribution to $\sigma_{x x}^{t o t}$ and $\sigma_{x y}^{t o t}$ from the coherent channel in Eq. (6). Note that similar reasoning does not hold for the incoherent sector because, by assumption, it contributes only to $\sigma_{x x}^{\text {inc }}$, while there is no contribution to $\sigma_{x y}^{i n c}$.

Since $f_{\sigma}<1$ and $n_{\mathrm{H}}(0)$ for $p<0.27$ is either at or below the dashed line in Fig. $1 \mathrm{~B}$ corresponding to the full Luttinger count, $n_{\text {coh }}$ must always be less than the total carrier density $n_{t o t}=1+p$ whenever there is a finite incoherent sector present. To reflect this, we introduce a second factor $f_{c o h}$ that represents the fraction of carriers that contribute to the coherent channel such that

$$
n_{\text {coh }}=f_{\text {coh }} n_{\text {tot }}=f_{\text {coh }}(1+p)
$$

From charge conservation, we can write

$$
n_{t o t}=n_{c o h}+n_{i n c}
$$


where $n_{\text {inc }}$ represents the 'missing' charge that contributes to $\sigma_{x x}^{i n c}$ but not to $\sigma_{x y}^{i n c}$

$$
n_{\text {inc }}=\left(1-f_{\text {coh }}\right) n_{\text {tot }}
$$

In this way, we establish how the incoherent sector manifests itself indirectly in both the Hall effect and resistivity data. In the following section, we proceed to fit the zero-field resistivity in order to obtain an estimate for $f_{\sigma}$ in OD Tl2201 for each doping level and from this, an estimate for $n_{c o h}(p)$.

\section{A.2 Fitting the zero-field resistivity}

Having introduced the weighting factors, we can now proceed to determine the separate contributions to the total (zero-field) conductivity in OD Tl2201. In total, five fitting parameters are required, two of them for the incoherent sector

$$
\rho_{x x}^{i n c}(T)=A+B T
$$

and three for the coherent sector

$$
\rho_{x x}^{c o h}(T)=C+D T+E T^{2}
$$

Note that both channels incorporate a finite residual resistivity in order to prevent $\rho(T=0)=0$. The linear $T$-dependence of $\rho_{x x}^{\text {inc }}(T)$ connects with the 'Planckian' quadrature MR reported in Ref. [16] and is assumed to be set by the Planckian dissipation limit

$$
\frac{\hbar}{\tau}=\alpha k_{B} T
$$

where $\alpha$ is of order unity.

The expression for $\rho_{x x}^{c o h}(T)$ contains both a Fermi-liquid quadratic term due to electronelectron scattering and an anomalous $T$-linear component. From analysis of the zerofield resistivity $\rho_{a b}(T)$ alone, it is difficult to ascertain whether the linear-in- $T$ component $(=D T)$ in the coherent channel is required since it turns out to be the most sensitive of all 5 parameters. Indeed, in some cases, it is possible to obtain reasonably good fits to $\rho_{a b}(T)$ with $D$ set to 0 . However, earlier analysis of $c$-axis angle-dependent magnetoresistance (ADMR) revealed the presence of two independent scattering channels in OD Tl2201; an isotropic $T^{2}$ scattering rate and an anisotropic $T$-linear scattering rate 67. As this was derived from analysis based on Boltzmann transport theory, it is assumed to reflect the behavior of the coherent sector. A $T$-linear component of orbital origin was also found to govern the temperature and field dependence of the Hall resistivity $\rho_{x y}(H)$ using the same parameterization derived from the ADMR experiments (for a similar doping) 14. Moreover, simultaneous fits of the in-plane MR of OD Tl2201 measured at different temperatures [16] found the magnitude of $B$ to be smaller than the total $T$-linear term observed in the absence of a magnetic field, a further indication that a fraction of the total $T$-linear component in $\rho_{x x}^{t o t}(T)$ originates from the coherent sector.

Finally, the experimentally-determined resistivity can be fitted to the following expression

$$
\rho_{x x}^{t o t}=\frac{\rho_{x x}^{c o h} \rho_{x x}^{i n c}}{\rho_{x x}^{c o h}+\rho_{x x}^{i n c}}
$$

which is nothing more than an inversion of Eq. (6). Examples are shown in Figure 3. Note that all fits were restricted to temperatures above which superconducting fluctuations were no longer evident. Typically, the zero-field resistivity was found to be insufficiently constrained to obtain fitted parameters that were insensitive to the initial fit conditions. However, some of the obtained values were unphysical (including negative residual resistivities 

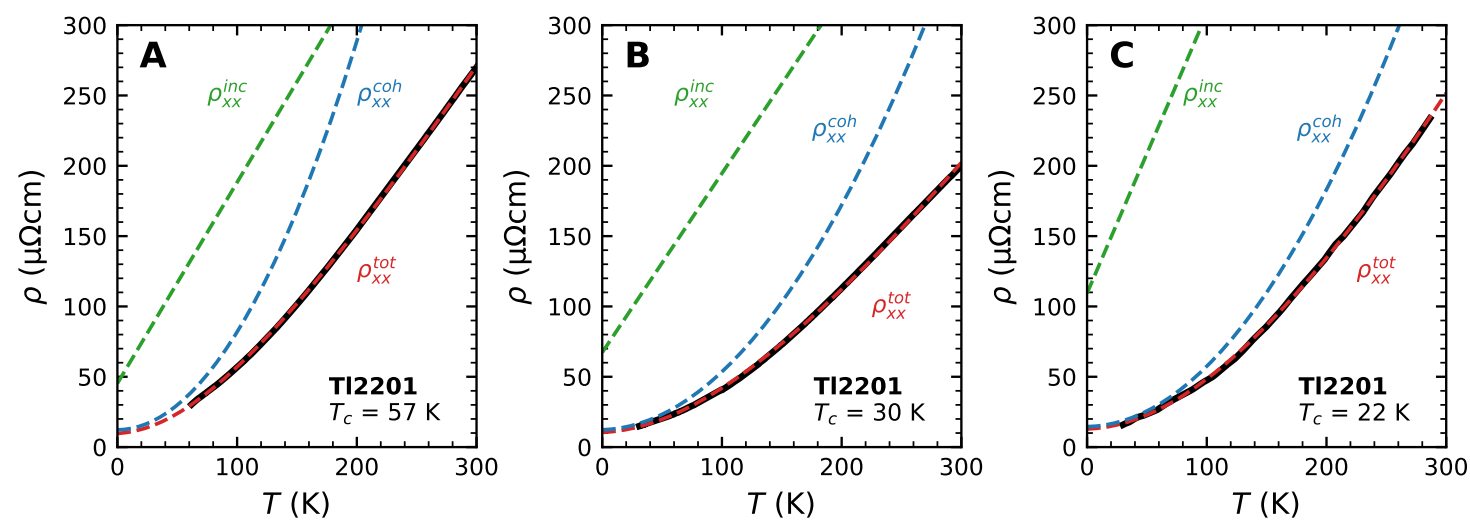

Figure 3: Parallel conductivity fits to the zero-field resistivities of OD Tl2201 with A): $T_{c}=57 \mathrm{~K}[13, \mathbf{B}): T_{c}=30 \mathrm{~K}[13]$ and $\left.\mathbf{C}\right): T_{c}=22 \mathrm{~K}[16$. Black lines represent the measured data, the blue dashed lines are the $\rho_{x x}^{c o h}(T)$ contributions described by Eq. (21) and the green dashed lines are the $\rho_{x x}^{i n c}(T)$ contributions described by Eq. (20). The red dashed line represents the resultant fit to $\rho_{x x}^{t o t}(T)$ as given by Eq. 23.

\begin{tabular}{|c|c|c|c|c|c|c|c|}
\hline $\begin{array}{c}T_{c} \\
(\mathrm{~K})\end{array}$ & $\begin{array}{c}\text { doping } p \\
( \pm 0.005)\end{array}$ & $A(\mu \Omega \mathrm{cm})$ & $\begin{array}{c}B \\
(\mu \Omega \mathrm{cm} / \mathrm{K})\end{array}$ & $C(\mu \Omega \mathrm{cm})$ & $\begin{array}{c}D\left(10^{-2}\right. \\
\mu \Omega \mathrm{cm} / \mathrm{K})\end{array}$ & $\begin{array}{c}E\left(10^{-3}\right. \\
\left.\mu \Omega \mathrm{cm} / \mathrm{K}^{2}\right)\end{array}$ & Ref. \\
\hline 57 & 0.235 & $45 \pm 8$ & $1.43 \pm 0.05$ & $12.2 \pm 6.1$ & $1.0 \pm 0.3$ & $6.9 \pm 0.8$ & {$[13$} \\
\hline 48 & 0.245 & $36 \pm 2$ & $1.57 \pm 0.07$ & $10.3 \pm 0.5$ & $5.3 \pm 0.3$ & $5.1 \pm 0.2$ & $\overline{13}$ \\
\hline 43 & 0.250 & $49 \pm 1$ & $1.61 \pm 0.13$ & $9.9 \pm 0.2$ & $1.0 \pm 0.2$ & $4.6 \pm 0.8$ & $\overline{13}$ \\
\hline 30 & 0.270 & $67 \pm 7$ & $1.27 \pm 0.14$ & $12.2 \pm 1.0$ & $2.6 \pm 0.3$ & $3.9 \pm 0.4$ & $\overline{13}$ \\
\hline 24 & 0.280 & $90 \pm 10$ & $1.20 \pm 0.05$ & $12.2 \pm 2.0$ & $2.4 \pm 0.2$ & $3.1 \pm 0.5$ & $\overline{16}$ \\
\hline 22 & 0.280 & $109 \pm 5$ & $2.00 \pm 0.17$ & $15.5 \pm 6.7$ & $1.5 \pm 0.7$ & $4.1 \pm 1.7$ & $\overline{16}$ \\
\hline 7 & 0.300 & $240 \pm 10$ & $26.1 \pm 2.2$ & $12.1 \pm 0.2$ & $1.3 \pm 0.4$ & $2.8 \pm 0.1$ & $\overline{13}$ \\
\hline
\end{tabular}

Table 1: Fitting parameters of the zero-field resistivities in the parallel conductivity channel scenario for T12201 13, 16. The parameters $A, B, C, D$ and $E$ are described in Eq. 20) and (21). The error bars indicate the standard deviation extracted from the fitting procedure. All parameters are also subject to an additional $20 \%$ error resulting from the uncertainty in sample geometry that is not included here but is incorporated into the error analysis in Fig. 1.

in variables $A$ and/or $C$ ), and some were found to be inconsistent with other experimental evidence (e.g. fits lacking a finite $D$ are inconsistent with the ADMR results). Bounds were set on some of the parameters in order to constrain the overall fitting procedure. These bounds were as follows: $\rho_{\text {upper }}<A<10 \mathrm{~m} \Omega \mathrm{cm}, 0.5 \mu \Omega \mathrm{cm} / \mathrm{K}<B, \rho_{\text {lower }}<C<\rho_{\text {upper }}$, $0.01 \mu \Omega \mathrm{cm} / \mathrm{K}<D, 1 \mathrm{n} \Omega \mathrm{cm} / \mathrm{K}^{2}<E$, where $\rho_{\text {lower }}$ is the (extrapolated) zero-temperature limit of the as-measured resistivity and $\rho_{\text {upper }}$ is a multiple of $\rho_{\text {lower }}$ chosen to reflect the expected decrease in the contribution from the incoherent sector to the total resistivity with increased doping. This prevented unphysical fits, for example, ones in which a sample with a low incoherent carrier density had a small incoherent resistivity. The bounds on $A$ and $C$ ensure that the incoherent sector is always more resistive that the coherent sector. Finally, it is, of course, unphysical to assume that the electron-electron scattering term in the expression for $\rho_{x x}^{c o h}(T)$ remains purely quadratic for all temperatures up to $300 \mathrm{~K}$. However, since the Planckian term in Eq. 20 effectively 'saturates' the $T$-linear slope of $\rho_{x x}^{t o t}(T)$ at high- $T$, its precise form is not expected to have any significant influence on the overall quality of the fits. 


\section{A.3 Coherent carrier density in $\mathrm{Tl}_{2} \mathrm{Ba}_{2} \mathrm{CuO}_{6+\delta}$}

Since our focus here is on the zero-temperature limit of both the normal and superfluid carrier densities, the relevant weighting factors $f_{\sigma}$ are determined from the ratio of the residual resistivities using the following expression derived from Eqs. (6), (13), (20) and (21)

$$
\frac{C}{A}=\frac{1-\sqrt{f_{\sigma}}}{\sqrt{f_{\sigma}}}
$$

Note that as this is a ratio of resistivities, any uncertainty in the absolute resistivity values is removed. Having obtained $f_{\sigma}$ using Eq. (24), we proceed to determine an estimate of the coherent carrier density $n_{c o h}$ for each doping level. The resultant values for $f_{\sigma}$ and $n_{\text {coh }}$ are listed in Table 2 and the doping dependence of $n_{\text {coh }}$ plotted as open circles in Fig. 4. The blue squares in Fig. 4 represent the Hall numbers $n_{\mathrm{H}}(0)$ for OD Tl2201 as determined by Putzke et al. [14]. As can be seen, recognition of the presence of the incoherent sector has led to a reduction in our estimate of the coherent carrier density for all doping levels. Thus, where it would appear from measurements of $n_{\mathrm{H}}(0)$ that the loss of coherent carriers begins at $p=0.27$, after accounting for the presence of the second charge sector, $n_{\text {coh }}(p)$ now appears to extrapolate to $1+p$ at $p \sim 0.31$, i.e., the doping at which superconductivity emerges.

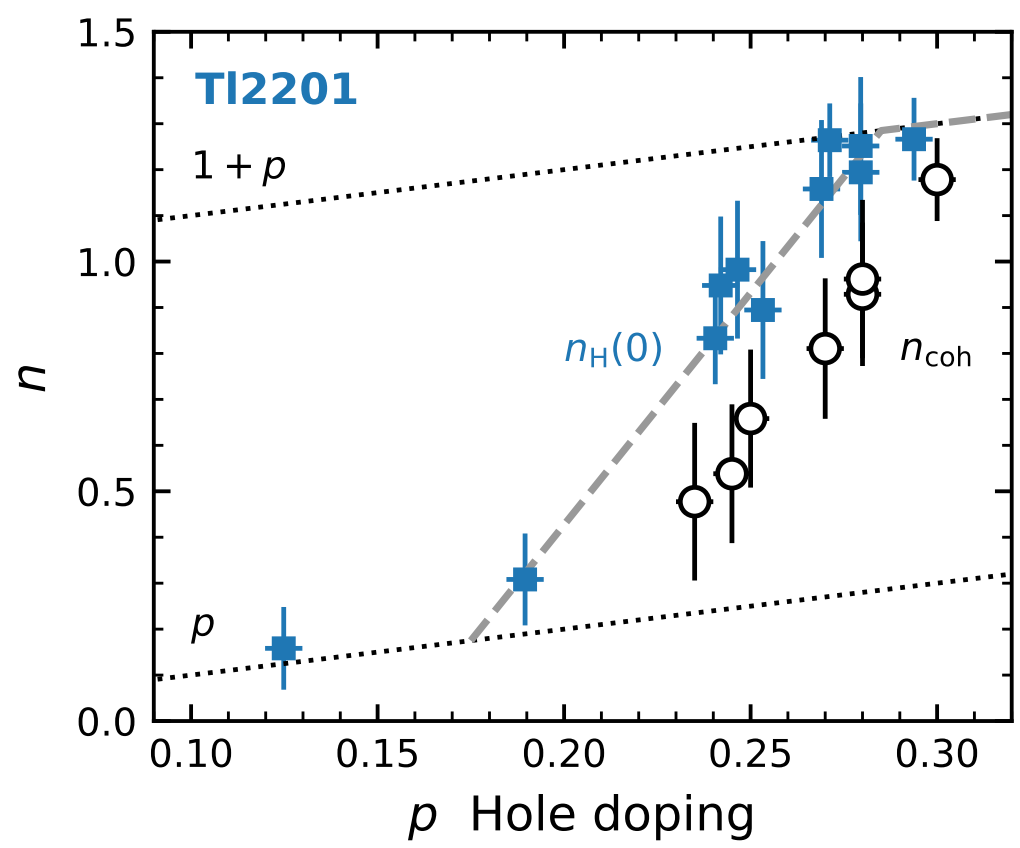

Figure 4: Measured Hall carrier density $n_{\mathrm{H}}(0)$ in OD Tl2201 [14] together with the coherent carrier density $n_{\text {coh }}$ in the zero-temperature limit extracted using Eq. (16). In order to obtain $n_{c o h}$, the Hall data [14] (blue squares) are first fitted to the faint dashed line $\left(n_{\mathrm{H}}(0)=0.175+10.1(p-0.175)\right)$. Then, for each $p$ value for which we have zero-field resistivity data, we obtain $f_{\sigma}$. Finally, multiplying the expression for $n_{\mathrm{H}}(0)$ by these $f_{\sigma}$ values, we obtain the open symbols corresponding to $n_{\text {coh }}$. The error bars for $n_{\text {coh }}$ were calculated as composite deviations coming from standard deviations in $f_{\sigma}$ and in $n_{\mathrm{H}}(0)$. 


\begin{tabular}{|c|c|c|c|c|c|}
\hline$T_{c}(\mathrm{~K})$ & $\begin{array}{c}\text { doping } p \\
( \pm 0.005)\end{array}$ & $n_{\mathrm{H}}(0)$ & $f_{\sigma}$ & $n_{\text {coh }}$ & Ref. \\
\hline 57 & 0.235 & $0.77 \pm 0.10$ & $0.62 \pm 0.14$ & $0.48 \pm 0.17$ & $\overline{13}$ \\
\hline 48 & 0.245 & $0.89 \pm 0.15$ & $0.60 \pm 0.02$ & $0.54 \pm 0.15$ & $\overline{13}$ \\
\hline 43 & 0.250 & $0.95 \pm 0.15$ & $0.69 \pm 0.01$ & $0.66 \pm 0.15$ & $\overline{\overline{13}}$ \\
\hline 30 & 0.270 & $1.13 \pm 0.15$ & $0.72 \pm 0.03$ & $0.81 \pm 0.15$ & $\overline{\overline{13}}$ \\
\hline 24 & 0.280 & $1.21 \pm 0.15$ & $0.76 \pm 0.04$ & $0.93 \pm 0.16$ & $\overline{\overline{16}}$ \\
\hline 22 & 0.280 & $1.23 \pm 0.15$ & $0.78 \pm 0.08$ & $0.96 \pm 0.17$ & $\overline{16}$ \\
\hline 7 & 0.300 & $1.30 \pm 0.09$ & $0.91 \pm 0.01$ & $1.18 \pm 0.09$ & $\overline{13}$ \\
\hline
\end{tabular}

Table 2: Extracted coherent carrier densities in the zero-temperature limit for different doping levels in OD Tl2201. The measured Hall number $n_{\mathrm{H}}(0)$ was determined for each doping level from the grey dashed line in Fig. 4 based on Ref. 14, while the weighting factor $f_{\sigma}$ was determined using Eq. (24) and the residual resistivity components listed in Table 1. Finally, Eq. (16) was used to obtain $n_{c o h}$. The error bars for $f_{\sigma}$ were calculated as composite deviations coming from standard deviations in resistivity parameters $A$ and $C$ and the error bars for $n_{c o h}$ were calculated as composite deviations coming from standard deviations in $f_{\sigma}$ and in $n_{\mathrm{H}}(0)$.

\section{A.4 Planckian dissipation in $\mathrm{Tl}_{2} \mathrm{Ba}_{2} \mathrm{CuO}_{6+\delta}$}

Having obtained the contributions of the individual sectors to the total conductivity, we examine here the resistivity of the incoherent sector and in particular, the magnitude of the $T$-linear relaxation rate $1 / \tau_{i n c}$ associated with $\rho_{x x}^{i n c}(T)$. As done previously [68,69], we assume a Drude form for $\sigma_{x x}^{i n c}$ and write

$$
A+B T=\frac{m^{*}}{n_{\text {inc }} e^{2} \tau_{\text {inc }}}
$$

Taking the derivative of Eq. 25) with respect to temperature and making use of Eq. (19), we find

$$
\frac{d\left(\hbar / \tau_{\text {inc }}\right)}{d T}=B\left(1-f_{\text {coh }}\right) \frac{\hbar n_{\text {tot }} e^{2}}{m^{*} V_{\text {cell }}}
$$

where $V_{\text {cell }}=173 \AA^{3}$ and $m^{*}=5.2 m_{e}$ (i.e., independent of doping), consistent with QO [29] and specific heat [30] measurements. (Note that the same approximation was used for determination of the superfluid density). The weighting factors $f_{\text {coh }}$ were determined using Eq. (17) and are listed in Table 3. In order to compare Eq. (26) with the Planckian expression (Eq. (22)), we set

$$
\frac{d\left(\hbar / \tau_{\text {inc }}\right)}{d T}=\alpha k_{B}
$$

Table 3 shows the resultant $\alpha$ values in Tl2201 for different doping levels. As we can see, for all the samples with $p<0.295$, the parameter $\alpha$ is between 1 and 3 , consistent with the value extracted in other cuprates 69 as well as many other correlated and quantum critical metals 68,70 . Hence, even though the magnitude of the $T$-linear coefficient in OD Tl2201 implies a scattering rate much smaller than the Planckian limit [13], incorporation of the two charge sectors into the analysis of the zero-field resistivity reveals that the scattering rate associated with the incoherent sector is itself Planckian.

\section{A.5 Validity of approximations used in Section A.1}

Here, we address the various approximations introduced in Section A.1 when fitting the zero-field resistivities to estimate the coherent and incoherent carrier densities. The first 


\begin{tabular}{|c|c|c|c|c|c|}
\hline$T_{c}(\mathrm{~K})$ & $\begin{array}{c}\text { doping } p \\
( \pm 0.005)\end{array}$ & $B(\mu \Omega \mathrm{cm} / \mathrm{K})$ & $f_{\text {coh }}$ & $\alpha$ & Ref. \\
\hline 57 & 0.235 & $1.43 \pm 0.05$ & $0.39 \pm 0.14$ & $2.78 \pm 0.64$ & 13 \\
\hline 48 & 0.245 & $1.57 \pm 0.07$ & $0.43 \pm 0.12$ & $2.85 \pm 0.62$ & $\overline{13}$ \\
\hline 43 & 0.250 & $1.61 \pm 0.13$ & $0.53 \pm 0.12$ & $2.45 \pm 0.65$ & $\overline{13}$ \\
\hline 30 & 0.270 & $1.27 \pm 0.14$ & $0.64 \pm 0.12$ & $1.50 \pm 0.52$ & $\overline{13}$ \\
\hline 24 & 0.280 & $1.20 \pm 0.05$ & $0.73 \pm 0.12$ & $1.08 \pm 0.48$ & $\overline{16}$ \\
\hline 22 & 0.280 & $2.00 \pm 0.17$ & $0.75 \pm 0.14$ & $1.62 \pm 0.89$ & $\overline{16}$ \\
\hline 7 & 0.300 & $26.1 \pm 2.2$ & $0.91 \pm 0.07$ & $8.1 \pm 6.1$ & 13 \\
\hline
\end{tabular}

Table 3: Estimate of the prefactor $\alpha$ in the $T$-linear resistivity term associated with the incoherent sector. $B$ is copied from Table 1 and $f_{c o h}$ is determined as described in the text. Parameter $\alpha$ is determined by comparing Eq. (26) and (27). The error bars for $f_{\text {coh }}$ were determined from the error bars in $n_{\text {coh }}$ and the error bars for $\alpha$ were calculated as composite deviations coming from standard deviations in $f_{c o h}$ and the resistivity parameter $B$. The high error bars for the lower $T_{c}$ samples arise due to the low value of $\left(1-f_{c o h}\right)$ used in Eq. 26. coupled with the uncertainty in $f_{\text {coh }}$.

of these, introduced in Eq. (10), assumes that for each sample, the longitudinal resistivity $\left(\rho_{x x}^{t o t}\right.$ or $\left.\rho_{x x}^{c o h}\right)$ far exceeds the corresponding Hall resistivity $\left(\rho_{x y}^{t o t}\right.$ or $\left.\rho_{x y}^{c o h}\right)$. The second, expressed in Eq. (11), is that $\rho_{x x}^{t o t} \approx \rho_{x x, 0}^{t o t}$ and $\rho_{x x}^{c o h} \approx \rho_{x x, 0}^{c o h}$. Much of our analysis described above is based on fitting of the zero-field resistivity and in the zero-field limit, of course, all these approximations become exact. The key question, therefore, is whether the values of $n_{\mathrm{H}}(0)$ extracted from the high-field Hall resistivity measurements of Ref. [14], are an accurate reflection of the $n_{\mathrm{H}}(0)$ values one would obtain in the absence of superconductivity. In Figure 1 of Ref. [14], the low- $T$ Hall coefficient $R_{\mathrm{H}}(0)\left(=\rho_{x y} / H\right)$ in Tl2201 crystals with $T_{c}$ values of $30 \mathrm{~K}$ and $40 \mathrm{~K}$ is found to be independent of field from the maximum field strength $(65 \mathrm{~T})$ down to $20 \mathrm{~T}$ and $30 \mathrm{~T}$ respectively. Below these field scales, each sample enters the vortex regime. Given the evolution with field and temperature in each sample, together with the simulations presented in Ref. [14] based on Boltzmann transport analysis, we are confident that these $R_{\mathrm{H}}(0)$ values are indeed representative of the low- $T$, low- $H$ Hall coefficients in OD Tl2201.

\section{A.6 Superfluid density in $\mathrm{Tl}_{2} \mathrm{Ba}_{2} \mathrm{CuO}_{6+\delta}$}

Table 4 lists the superfluid densities of OD Tl2201 samples measured via muon-spin relaxation $(\mu \mathrm{SR})$ measurements on polycrystalline [4,5] and single crystalline [28] samples and a microwave surface impedance measurement carried out on a Tl2201 single crystal with $T_{c} \approx 25 \mathrm{~K}[6]$. Excellent agreement is found between the various data sets. The doping levels quoted in Table 4 are obtained using the linear $T_{c}(p)$ dependence deduced from previous quantum oscillation studies [29], while the superfluid density values $n_{s}(0)$ are obtained using

$$
n_{s}(0)=\frac{m^{*} V_{\text {cell }}}{\mu_{0} e^{2} \lambda_{a b}^{2}(0)}
$$

where $\lambda_{a b}(0)$ is the in-plane zero-temperature penetration depth and again, it is assumed that $m^{*}=5.2 m_{e}$ throughout. In order to obtain $1 / \lambda_{a b}^{2}(0)$ for the Uemura data [4] where only values of the depolarization rate $\sigma$ were quoted, we used the relation from Niedermayer et al. 5]

$$
\sigma\left[\mu \mathrm{s}^{-1}\right]=7.086 \cdot 10^{4} \cdot 1 / \lambda_{a b}^{2}(0)[\mathrm{nm}]
$$




\begin{tabular}{|c|c|c|c|c|}
\hline$T_{c}(\mathrm{~K})$ & doping $p$ & $1 / \lambda_{a b}^{2}(0)\left(\mu \mathrm{m}^{-2}\right)$ & $n_{s}(0)$ & Ref. \\
\hline 60 & 0.23 & $32.7 \pm 0.3$ & $0.78 \pm 0.01$ & $\overline{28}$ \\
\hline 56 & 0.235 & $36.3 \pm 0.4$ & $0.86 \pm 0.01$ & $\overline{28}$ \\
\hline 53 & 0.24 & $28.9 \pm 1.8$ & $0.69 \pm 0.04$ & $\overline{[5}$ \\
\hline 50 & 0.245 & $32.5 \pm 1.7$ & $0.77 \pm 0.04$ & $\overline{4}$ \\
\hline 46 & $0.25(1)$ & $28.6 \pm 0.3$ & $0.68 \pm 0.01$ & $\overline{28}$ \\
\hline 46 & $0.25(2)$ & $36.7 \pm 0.4$ & $0.87 \pm 0.01$ & $\overline{28}$ \\
\hline 25 & 0.275 & $14.6 \pm 1.5$ & $0.35 \pm 0.04$ & $\overline{6}$ \\
\hline 20 & 0.285 & $14.1 \pm 0.7$ & $0.33 \pm 0.02$ & $\overline{4}$ \\
\hline 13 & 0.295 & $7.8 \pm 1.6$ & $0.19 \pm 0.04$ & $\overline{5}$ \\
\hline
\end{tabular}

Table 4: Estimated superfluid density $n_{s}(0)$ in OD Tl2201 as a function of doping, derived from measurements of muon-spin relaxation [4,5,28] and microwave surface impedance [6]. The $T_{c}$ values are taken from the references. The corresponding $p$ values are obtained using the linear relation for $T_{c}(p)$ derived from quantum oscillation experiments [29]. Uncertainties for $p=0.230,0.235,0.25(1), 0.25(2)$ are quoted in Ref. [6]; uncertainties for $p=0.275$ are quoted in Ref. [28]. Errors for $p=0.24$ and 0.295 were obtained through the standard deviation of a linear fit to all four samples in Fig. 1 of Ref. [5]. Errors for $p=0.245$ and 0.285 were found through the uncertainty in the zero-temperature relaxation rate $\sigma_{0}$ fitting the data in Fig. 1(b) of Ref. [4] to the form $\sigma_{0}\left[1-\left(T / T_{c}\right)^{\alpha}\right]$ where $\alpha \leq 458$.

The resultant doping dependences of the superfluid, normal and total carrier densities in OD Tl2201 are presented in Fig. 1 .

\section{B Obtaining estimates for $n_{c o h}$ and $n_{s}(0)$ in $\mathrm{La}_{2-x} \mathrm{Sr}_{x} \mathrm{CuO}_{4}$}

In this section, we show how $n_{c o h}$ and $n_{s}(0)$ are obtained for overdoped LSCO. The derivation of both requires more careful analysis than for Tl2201 due to the vicinity of the vHs in OD LSCO and the resultant strong anisotropy in the FS and in the low- $T$ meanfree-path $\ell_{0}(\mathbf{k})$. Nevertheless, LSCO remains to date the only cuprate beside Tl2201 for which sufficiently detailed Hall and superfluid density data are available to perform a similar analysis to that presented in Appendix A. Furthermore, these differences between LSCO and Tl2201 warrant a parallel study in order to verify whether or not the observed effects generalize across different cuprate families.

\section{B.1 Hall effect in $\mathrm{La}_{2-x} \mathrm{Sr}_{x} \mathrm{CuO}_{4}$}

In contrast to Tl2201, obtaining $n_{\mathrm{H}}(0)$ in OD LSCO from measurements of the limiting low- $T$ Hall coefficient $R_{\mathrm{H}}(0)$ is non-trivial. In this section, we address the pertinent issues and use the existing parameterization of the LSCO FS as well as knowledge of $\ell(\mathbf{k})$ to demonstrate why it is still necessary to invoke the presence of an incoherent channel in OD LSCO (more precisely, a region of the FS in which $\sigma_{x y}=0$ ) to obtain a reliable estimate of $R_{\mathrm{H}}(0)$ and from this, obtain estimates for $n_{\mathrm{s}}(0)$ and $n_{c o h}$ across the entire OD regime.

According to ARPES measurements, the quasi-2D FS in LSCO undergoes a Lifshitz transition around $x=p=0.195$ when the Fermi level crosses the vHs near $(\pi, 0)$. Hence, beyond $x=0.195$, the FS possesses both electron- and hole-like curvature, the former near the zone boundary and the latter near the zone diagonals (shown in Fig. 5 for three 


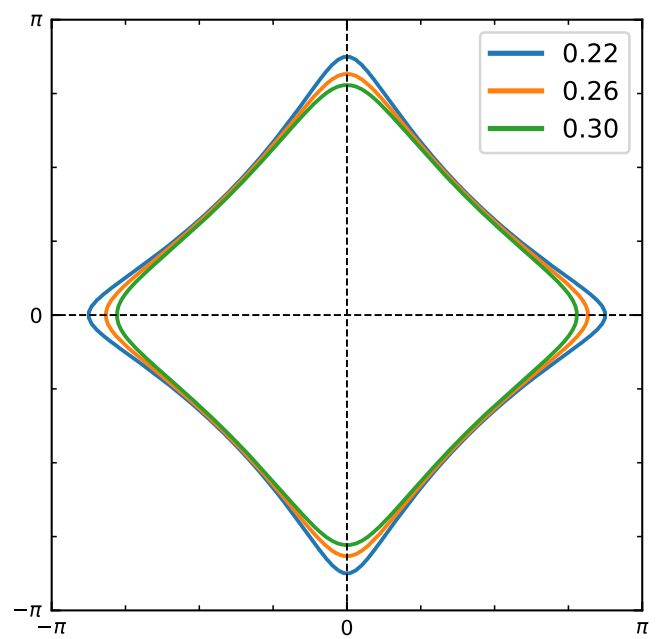

Figure 5: Tight binding parameterization for three dopings, $p=0.22,0.26,0.30$ in OD LSCO based on ARPES spectra by Yoshida [33. The FS contains both electron- and holelike curvature which, coupled with an anisotropy in $\ell(\mathbf{k})$ at low- $T$, leads to contributions to $\sigma_{x y}$ of different sign.

\begin{tabular}{|c|c|c|c|c|}
\hline doping $p$ & $e_{0}$ & $t_{0}$ & $t_{1}$ & $t_{2}$ \\
\hline 0.16 & 0.2025 & 0.2500 & -0.0375 & 0.0188 \\
\hline 0.185 & 0.2080 & 0.2500 & -0.0355 & 0.0177 \\
\hline 0.19 & 0.2096 & 0.2500 & -0.0350 & 0.0175 \\
\hline 0.21 & 0.2144 & 0.2500 & -0.0338 & 0.0169 \\
\hline 0.26 & 0.2295 & 0.2500 & -0.0312 & 0.0156 \\
\hline
\end{tabular}

Table 5: Tight-binding parameters from Lee-Hone et al. 63

separate doping levels). A strong variation of the in-plane Fermi velocity $v_{F}$ then arises due to the presence of the closely-lying saddle points. In conventional metals, anisotropy in $v_{F}(\mathbf{k})$ is usually compensated for by anisotropy in $\tau(\mathbf{k})$ and as a result, $\ell_{0}$ becomes isotropic at zero temperature (the average distance between impurities within the conducting plane being independent of direction). In LSCO, however, it has been argued [36 that the scattering rate at a given momentum depends on the local density of states due to the fact that the ( $\mathrm{Sr}$ ) dopant impurities lie outside of the $\mathrm{CuO}_{2}$ plane. This anisotropy in $1 / \tau(\mathbf{k})$ in turn amplifies, rather than nullifies, any anisotropy in $v_{F}(\mathbf{k})$, leading to a marked violation of the isotropic- $\ell$ approximation. This violation then leads to a complicated expression for the low-field $R_{\mathrm{H}}(0)$ which does not directly reflect the carrier density. The anisotropy in $\ell_{0}$, defined as the ratio $\beta=\ell(\pi, \pi) / \ell(\pi, 0)$, has been derived in both Hall effect [34] and ADMR 35] measurements and is found to be substantial, rising from $\beta \sim 10$ at $p=0.33$ 34 to $\beta \sim 100$ at $p=0.24$ (in Nd-LSCO) 35].

An elegant geometrical interpretation of the weak-field Hall conductivity $\sigma_{x y}$ in $2 \mathrm{D}$ metals was introduced by Ong in 1991 [45. In metals with a FS that possesses both negative FS curvature (i.e. sections of opposing circulation of the $\ell$-vector as it is swept around the FS) and anisotropy in $\ell(\mathbf{k}), \sigma_{x y}$ is given by the integral of the 'Stokes' area $A=\int(d \ell \times \ell) / 2$ over the full $2 \mathrm{D}$ FS. The corresponding ' $\ell$-curve' contains areas with opposite circulation and therefore contributions to $\sigma_{x y}$ of opposite sign. A schematic 

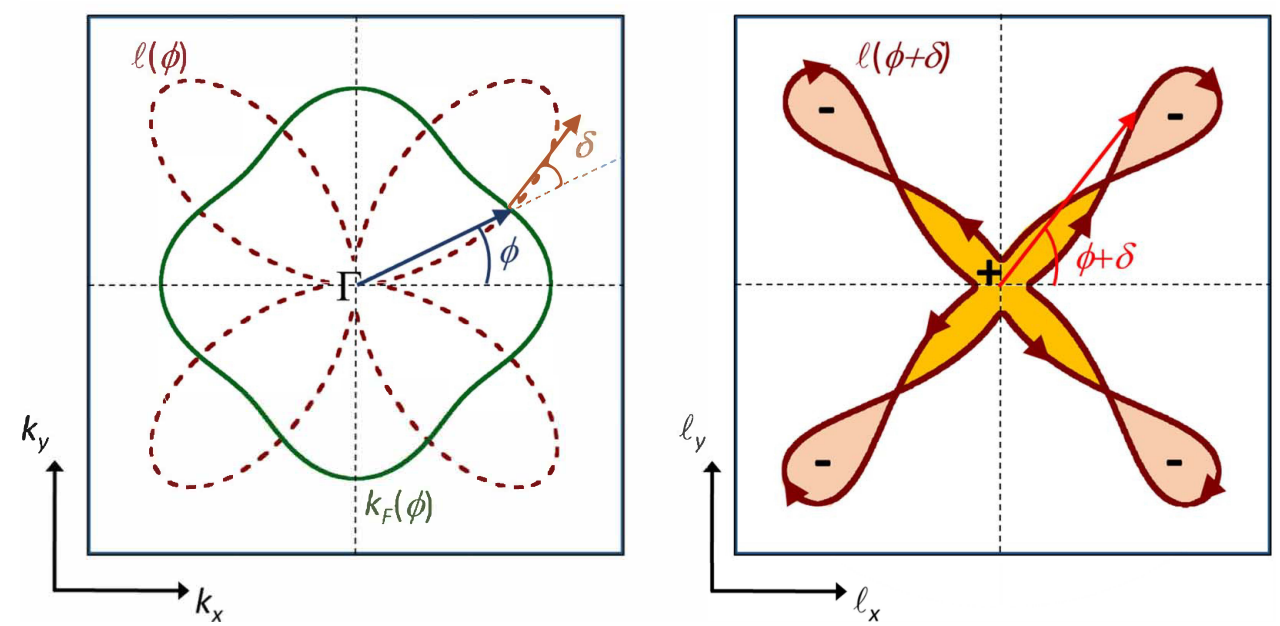

Figure 6: Left panel: Section of a 2D Fermi surface with pronounced negative curvature. The solid green line indicates the Fermi surface $k_{F}(\phi)$ and the brown dashed line indicates a strongly anisotropic mean free path $\ell(\phi)$. The blue arrow indicates the direction and length of $k_{F}$ while the orange arrow indicates the direction and length of $\ell$. Right panel: Polar plot of $\ell(\phi)$. The tangential arrows indicate the circulation of each loop and the $-/+$ signs indicate the corresponding sign of the loop. The red arrow again indicates the direction and length of $\ell$. The resultant $\sigma_{x y}$ is determined by the total area, i.e. the difference in the areas of the two counter-rotating loops.

example of this is given in Fig. 6 for a FS geometry similar to that realized in OD LSCO. The conductivity, $\sigma_{x x}$ is determined from the appropriate integral, and the corresponding Hall coefficient through $R_{\mathrm{H}}=\sigma_{x y} / \sigma_{x x}^{2}$. Application of the Ong representation to heavily OD, non-superconducting LSCO $(x=0.33)$, using FS information derived from ARPES [33, was found to reproduce both $R_{\mathrm{H}}(0)$ and its $T$-dependence up to $300 \mathrm{~K}$ 34. Note that in this case, the full FS was included in the calculation of $R_{\mathrm{H}}(0)$ while $\beta \approx 10$.

\begin{tabular}{|c|c|}
\hline doping $p$ & Correction Factor \\
\hline 0.20 & $1.000 \pm 0.050$ \\
\hline 0.21 & $1.005 \pm 0.050$ \\
\hline 0.22 & $1.010 \pm 0.051$ \\
\hline 0.23 & $1.025 \pm 0.051$ \\
\hline 0.24 & $1.040 \pm 0.052$ \\
\hline 0.25 & $1.055 \pm 0.053$ \\
\hline 0.26 & $1.065 \pm 0.053$ \\
\hline 0.275 & $1.075 \pm 0.054$ \\
\hline 0.3 & $1.085 \pm 0.054$ \\
\hline 0.32 & $1.115 \pm 0.056$ \\
\hline
\end{tabular}

Table 6: Correction factor for the angle $\delta$ between $k_{F}$ and $v_{F}$; the uncertainty indicates the spread of values providing a Fermi surface which fits within the width of the ARPES resolution.

In order to explore whether the measured values of the Hall coefficient can be reproduced within the Ong representation for other dopings, we calculated $R_{\mathrm{H}}(0)$ over the entire overdoped regime using the ARPES-derived FS parameterizations of Yoshida et al. [33. (Note that all subsequent ARPES studies [71 74] have found FS geometries that are consistent with the parameterizations reproduced in Fig. 5). It should be empha- 
sized here that while the doping evolution of the FS appears slight, proximity to the vHs dictates that changes in the density of states and therefore in $\ell(\phi)$ around the FS have a strong doping dependence. Tight binding (TB) parameters are given in Table 5 . The area enclosed by the TB-derived Fermi surfaces was found to correspond invariably to a FS of higher doping. Thus, a correction was made by increasing the Fermi wave vector $k_{F}$ until $\left(1-2 \times \frac{\text { Area FS }}{\text { Area BZ }}\right)=1+p$ where $p$ is the doping. To better fit the ARPES data of Yoshida et al. [33 for $p=0.22$ and $p=0.30$, the curvature of the FS was also altered by modifying slightly the angle $\delta$ between $k_{F}$ and $v_{F}$ while maintaining the correct Luttinger count. The multiplication factor was interpolated for intermediate dopings and the full set listed in Table 6. Examples of uncorrected and corrected Fermi surfaces are shown for $p=0.22$ and $p=0.30$ in Figures $7 \mathrm{~B}$ and $7 \mathrm{C}$. The effect of these corrections was to increase slightly the modelled value of $R_{\mathrm{H}}(0)$. For $p=0.30$, the correction ensured an excellent match between the experimental and calculated Hall coefficient. It is important to note that variation in $\delta$ alone is not sufficient to fully renormalise $R_{\mathrm{H}}(0)$ to the literature values for all $p<0.27$. The green and blue dashed lines in Fig. $7 \mathrm{~A}$ represent the evolution of the calculated $R_{\mathrm{H}}(0)$ values based on the corrected and uncorrected FS parameterizations, respectively. The inverse scattering rate $\tau^{-1}(\mathbf{k})$ takes the Abrahams-Varma form [36] using the anisotropy of the TB-derived $v_{F}(\phi)$ multiplied by a constant chosen to obtain a residual resistivity $\rho_{x x}(0)=20 \mu \Omega \mathrm{cm}$ (a typical value in OD LSCO) in the fully coherent case. The anisotropy is reflected in $\ell(\mathbf{k})$ through $\ell(\mathbf{k})=v_{F}(\mathbf{k}) \tau(\mathbf{k})$.
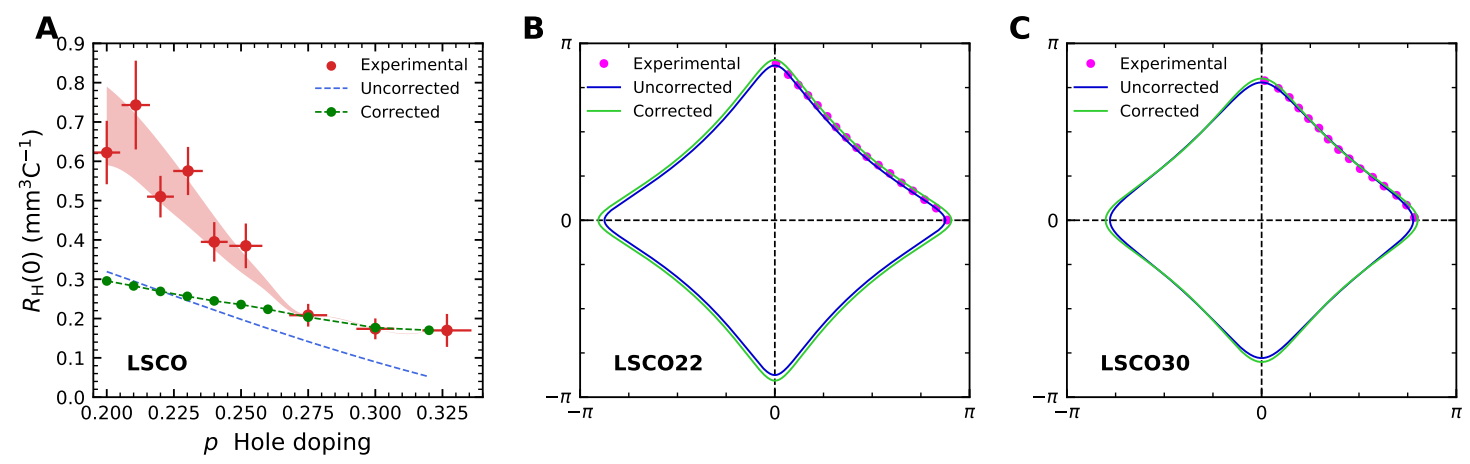

Figure 7: A): Doping dependence of the low- $T R_{\mathrm{H}}(0)$ in OD LSCO. (Red circles) Binned and averaged $R_{\mathrm{H}}(0)$ values obtained from the literature (see Table 7 and text for details of how these were obtained). The red shaded area provides an indication of the spread in the (binned and averaged) experimental values. The blue line is the evolution of $R_{\mathrm{H}}(0)$ estimated from Boltzmann transport theory using the TB parameterization of the (full) FS and assuming a scattering rate $1 / \tau(\phi)$ with the same in-plane anisotropy as the ARPESderived $v_{F}(\phi)$. The green circles are the values of $R_{\mathrm{H}}(0)$ calculated again assuming a fully coherent FS but now with a correction to satisfy the Luttinger count and adjust the FS curvature accordingly (but still remain consistent with the ARPES measurements to within their experimental momentum resolution). The green dashed line is an interpolation between these points. Note the bifurcation of the red symbols and the green dashed line at $p=p_{S C}=0.27$. Error bars for the experimental $R_{\mathrm{H}}(0)$ values are obtained from the standard deviation of the binned literature values coupled with an estimated $10 \%$ uncertainty in the determination of the sample thicknesses. B): Comparison between the uncorrected (blue) and corrected (green) Fermi surfaces for $p=0.22$ compared with the locus (solid fuchsia circles) of the ARPES-derived FS from Yoshida et al. [33. C): Same comparison for $p=0.30$. In both cases, the change in curvature matches well with the ARPES FS; for $p=0.30$, the corrected and as-measured $R_{\mathrm{H}}(0)$ values are found to agree. 


\begin{tabular}{|c|c|c|c|c|c|c|}
\hline $\begin{array}{c}\text { doping } \\
p\end{array}$ & $T_{c}(\mathrm{~K})$ & $\begin{array}{c}R_{\mathrm{H}}(0) \\
\left(\mathrm{mm}^{3} / \mathrm{C}\right)\end{array}$ & $\begin{array}{c}R_{\mathrm{H}}^{\max } \\
\left(\mathrm{mm}^{3} / \mathrm{C}\right)\end{array}$ & $\begin{array}{c}Z= \\
R_{\mathrm{H}}^{\max } / R_{\mathrm{H}}(0)\end{array}$ & $\begin{array}{c}R_{\mathrm{H}}^{*}(0)= \\
R_{\mathrm{H}}^{\max } / Z_{\text {fit }} \\
\left(\mathrm{mm}^{3} / \mathrm{C}\right)\end{array}$ & Ref. \\
\hline 0.20 & & & 0.70 & & 0.72 & $\overline{75}$ \\
\hline 0.20 & & 0.67 & 0.78 & 1.16 & 0.63 & $\overline{76}$ \\
\hline 0.21 & 27 & 0.83 & 0.96 & 1.16 & 0.79 & $\overline{77}$ \\
\hline 0.21 & & 0.60 & 0.78 & 1.29 & 0.63 & $\overline{778}$ \\
\hline 0.21 & 26 & & 0.88 & & 0.59 & $\overline{779}$ \\
\hline 0.22 & & 0.50 & 0.62 & 1.26 & 0.52 & $\overline{76}$ \\
\hline 0.23 & 20 & 0.56 & 0.70 & 1.25 & 0.58 & $\overline{80}$ \\
\hline 0.23 & & & 0.65 & & 0.54 & $\overline{81}$ \\
\hline 0.23 & 21 & 0.60 & 0.70 & 1.17 & 0.58 & $\overline{77}$ \\
\hline 0.235 & 18.5 & & 0.70 & & 0.39 & $\overline{779}$ \\
\hline 0.24 & 16 & 0.34 & & & 0.34 & $\overline{777}$ \\
\hline 0.24 & & 0.45 & 0.45 & 1.13 & 0.45 & $\overline{82}$ \\
\hline 0.25 & & & 0.52 & & 0.44 & $\overline{81}$ \\
\hline 0.25 & & 0.35 & 0.42 & 1.20 & 0.36 & $\overline{76}$ \\
\hline 0.255 & 7.5 & & 0.46 & & 0.13 & $\overline{77}$ \\
\hline 0.275 & 0 & 0.20 & 0.25 & 1.25 & 0.22 & $\overline{76}$ \\
\hline 0.30 & 0 & 0.17 & 0.20 & 1.18 & 0.18 & $\overline{76}$ \\
\hline 0.32 & 0 & & 0.14 & & 0.72 & $\overline{779}$ \\
\hline 0.33 & 0 & 0.20 & 0.20 & 1.00 & 0.18 & $\overline{334}$ \\
\hline
\end{tabular}

Table 7: Experimental values for $R_{\mathrm{H}}(0)$ for OD LSCO obtained from the literature. In samples where $T_{c}$ values were reported, the $p$ values were obtained using the standard parabolic $T_{c}(p)$ relation [83. In others, the $p$ values are as given. Low- $T$ values $R_{\mathrm{H}}(0)$, where reported, are listed. For those where only maximum $R_{\mathrm{H}}$ values $\left(=R_{\mathrm{H}}^{\max }\right)$ were plotted, $R_{\mathrm{H}}(0)$ values were estimated by fitting the ratios $Z=R_{\mathrm{H}}^{\max } / R_{\mathrm{H}}(0)$ of the other samples as a function of $p$, then using the (linear) fit to this ratio to estimate $R_{\mathrm{H}}(0)^{*}$ from $R_{\mathrm{H}}^{\max } / Z_{f i t}$. Using the same fit to 're-engineer' $R_{\mathrm{H}}(0)$ for those samples for which $R_{\mathrm{H}}(0)$ was already known shows that the fitting routine reproduced the as-measured $R_{\mathrm{H}}(0)$ values to within $10 \%$. All extracted values for $R_{\mathrm{H}}(0)$ and $R_{\mathrm{H}}(0)^{*}$ were subsequently binned into $p$ steps of 0.01 (or higher) and plotted in Fig. 7.

Fig. 7 presents a summary of the experimentally-determined values for $R_{\mathrm{H}}(0)$ in OD LSCO as reported in Ref. 34, 75, 82 together with others estimated from the maximum in $R_{\mathrm{H}}(T)$. The reported values as well as the procedure for estimating $R_{\mathrm{H}}(0)$ from $R_{\mathrm{H}}(T)$ are presented in Table 7. The red shaded region reflects the spread in the (binned and averaged) literature values in OD LSCO. The green circles are the calculated $R_{\mathrm{H}}(0)$ values and the green dashed line is an interpolation between these points. While for $p>0.27$, the calculated $R_{\mathrm{H}}(0)$ values assuming a full coherent FS agree extremely well with the as-measured values (in accordance with Ref. [34]), there is a clear bifurcation of the calculated and experimental values at $p=p_{S C}=0.27$, i.e. precisely at the point where superconductivity emerges in heavily OD LSCO.

\section{B.2 Estimating the superfluid density in $\mathrm{La}_{2-x} \mathrm{Sr}_{x} \mathrm{CuO}_{4}$}

We begin by considering the residual electronic specific heat coefficient $\gamma(0)$ which is observed in the superconducting state but whose origin is not yet qualitatively understood [38, 84, 85. . Using Eq. (4) from the main text as well as the TB-derived FS parameters, 


\begin{tabular}{|c|c|c|}
\hline doping $p$ & $v_{F} / v_{F}^{0}$ & Reference \\
\hline 0.20 & 1.96 & {$[\overline{7}$} \\
\hline 0.21 & 2.27 & $\overline{37}$ \\
\hline 0.21 & 2.27 & $\overline{7}$ \\
\hline 0.22 & 2.46 & $\overline{7}$ \\
\hline 0.23 & 2.56 & $\overline{7}$ \\
\hline 0.24 & 2.60 & $\overline{37}$ \\
\hline 0.24 & 2.60 & $\overline{7}$ \\
\hline 0.26 & 2.53 & $\overline{7}$ \\
\hline 0.27 & 2.46 & $\overline{37}$ \\
\hline 0.30 & 2.15 & - \\
\hline 0.32 & 1.71 & - \\
\hline
\end{tabular}

Table 8: Renormalisation factors for the Fermi velocity of LSCO where $v_{F}^{0}$ is taken from ARPES-derived tight-binding Fermi surfaces [33] and $v_{F}$ is the Fermi velocity required to match the as-measured normal-state electronic specific heat $\gamma_{N}[38,42,44]$.

we first calculate $\gamma_{N}$ (the normal state electronic specific heat coefficient) by integrating over the entire FS. As mentioned in the main text, there is a discrepancy of order 2 in the measured values relative to the calculated values. Table 8 shows the actual scaling parameter across the full doping range.

To proceed, we introduce the second (incoherent) charge sector as done in Section A for Tl2201. We first adopt scenario $I$ and assume that the coherent states reside near the nodal points (i.e. where the $d$-wave superconducting order parameter vanishes) along $(\pi, \pi)$ while the incoherent states reside near the 'anti-nodes' near $(\pi, 0)$, as inferred from ARPES measurements within the strange metal regime [71]; the converse scenario (i.e. scenario $C$ with the incoherent states at the nodes) is considered at the end of section B.3. In order to simplify the subsequent calculations, the boundary between the two sectors is assumed to be sharp. In the following, we refer to the remaining coherent part of the FS as the truncated FS. Note that this truncation is conceptually different to what has been inferred previously for the pseudogap state, where, according to ARPES, the FS is truncated into disconnected Fermi arcs separated by regions where there is negligible spectral weight at the Fermi level. In our model, the states in those regions indicated by the green dashed lines in Figure 2A remain at the Fermi level (and thus contribute to $\gamma_{N}$, but nonetheless display incoherent non-FL transport behaviour).

Using Eq. (5), we first calculate $1 / \lambda_{a b}^{2}(0)$ for the full FS (using the renormalised $v_{F}$ values) and truncate the FS integral until the calculation matches the as-measured value. The amount of truncation required then sets the coherent-incoherent boundary used in all subsequent calculations. Values for $1 / \lambda_{a b}^{2}(0)$ and $n_{\mathrm{s}}(0)$ are shown in Table 9. With the same coherent-incoherent boundary, $\gamma(0)$ is obtained from Eq. (4) from the appropriately truncated integral. The resultant ratios $\gamma(0) / \gamma_{N}$ are plotted in Fig. 2D and compared with the experimental data summarized in Ref. [38,39] as well as with the Knight shift results of Ref. [40]. A scenario in which the FS is divided into two distinct charge sectors is thus found to account well for the observed doping dependence of the uncondensed carriers.

\section{B.3 Coherent carrier density in $\mathrm{La}_{2-x} \mathrm{Sr}_{x} \mathrm{CuO}_{4}$}

Having established the degree of truncation of the FS to match $1 / \lambda_{a b}^{2}(0)$ to experiment, we now return to the discrepancy between the as-measured Hall coefficient and the modelled values for the fully coherent FS revealed in Fig. 77A. We again proceed by assuming that the 


\begin{tabular}{|c|c|c|c|c|c|}
\hline doping $p$ & $1 / \lambda_{a b}^{2}{ }^{\text {meas }}\left(\mu \mathrm{m}^{2}\right)$ & $1 / \lambda_{a b}^{2 \text { full }}\left(\mu \mathrm{m}^{2}\right)$ & $1 / \lambda_{a b}^{2 \text { trunc }}\left(\mu \mathrm{m}^{2}\right)$ & $n_{s}(0)$ & Reference \\
\hline 0.20 & 16.70 & 69.67 & 16.67 & 0.581 & {$[\overline{7}$} \\
\hline 0.21 & 20.30 & 59.63 & 20.25 & 0.697 & $\overline{37}$ \\
\hline 0.21 & 14.90 & 59.63 & 14.85 & 0.570 & $\overline{7}$ \\
\hline 0.22 & 12.60 & 54.19 & 12.55 & 0.521 & {$[\overline{7}$} \\
\hline 0.23 & 10.20 & 52.03 & 10.18 & 0.447 & $\overline{7}$ \\
\hline 0.24 & 11.10 & 50.97 & 11.06 & 0.468 & $\overline{37}$ \\
\hline 0.24 & 7.00 & 50.97 & 6.99 & 0.325 & $\overline{7}$ \\
\hline 0.25 & 3.60 & 51.43 & 3.61 & 0.170 & $\overline{7}$ \\
\hline 0.26 & 0.70 & 51.83 & 0.68 & 0.098 & $\overline{7}$ \\
\hline 0.27 & 0.15 & 51.89 & 0.00 & 0.030 & $\overline{37}$ \\
\hline 0.3 & 0.00 & 59.39 & 0.00 & 0.000 & - \\
\hline 0.32 & 0.00 & 73.39 & 0.00 & 0.000 & - \\
\hline
\end{tabular}

Table 9: Measured and calculated $1 / \lambda_{a b}^{2}(0)$ values for a full and truncated FS in OD LSCO. $n_{s}(0)$ is the estimated superfluid density obtained as described in the text. For $p=0.27$, the model was unable to reach the experimentally determined penetration depth. Thus, $n_{s}(0)$ is calculated using the London equation with $m^{*}=8.2$ where $m^{*}$ is estimated from the as-measured electronic specific heat [7, 37]. Obviously, no penetration depth results have been reported on the non-SC samples but they are included here for completeness.

incoherent sector has a longitudinal conductivity that is additive but a Hall conductivity that is zero. We then perform the Ong construction for $\sigma_{x y}$ but with the integral truncated by the boundaries defined in section B.2. The precise expression for $\sigma_{x y}$ (with the full integration limits) is [46]:

$$
\sigma_{x y}=-\frac{e^{2} \mu_{0} H}{2 \pi^{2} \hbar^{2} d} \int_{0}^{2 \pi} \ell_{x}(\phi) \frac{\partial}{\partial \phi} \ell_{y}(\phi) d \phi
$$

The variation in $\ell(\phi)$ and in $k_{F}(\phi)$ used in the above expression is determined as described above. All symbols have their usual meanings, $\mu_{0} H=1 \mathrm{~T}$, and $d=6.6 \AA$ is the interplanar distance. The fraction of coherent FS, $f_{c o h}$, is simply given by the ratio 1 - $n_{s}(0) /(1+p)$. The calculated $R_{\mathrm{H}}(0)$ values are listed in Table 10 and plotted in Figures $2 \mathrm{~B}$ and $2 \mathrm{C}$, respectively. As can be seen in Figure $2 \mathrm{C}$, the truncated values of $R_{\mathrm{H}}(0)$ are within $0.2 \mathrm{~mm}^{3} / \mathrm{C}$ of the experimental value and the distinct bifurcation from the full FS calculation at $p_{S C}=0.27$ is well captured by the simulation.

In order to test the validity of our assumption that incoherent transport derives from carriers located at the anti-nodal regions of the FS, the above calculations were repeated with inverted integral boundaries (scenario $C$ ), i.e. the coherent and incoherent sectors positioned at the anti-nodes and nodes respectively. For all dopings within the superconducting dome, $\gamma(0) / \gamma_{N}$ showed no increase with doping and the resulting $R_{\mathrm{H}}(0)$ became negative (the region of the FS with positive curvature now having been truncated out) with a value that is one order of magnitude larger than the experimental values. In addition, the calculated incoherent carrier densities conflicted with values inferred from superfluid density measurements, as shown in Table 11. Thus, 'scenario $C$ ' was deemed to be inappropriate for OD LSCO.

On empirical grounds, one could further imagine that incoherence affects $\sigma_{x y}$ isotropically. In order to explain an increase in $R_{\mathrm{H}}(0)=\sigma_{x y} / \sigma_{x x}^{2}$ by a factor $2-3$, as indicated in Fig. $2 \mathrm{C}$, a sizable drop in $\sigma_{x x}$ would be required. However, such a decrease is not observed in the model where $\sigma_{x x}$ remains comparatively unaltered. Thus, an anisotropic change in the Hall conductivity is required. Combined with the above, we conclude that preferential 
suppression of $\sigma_{x y}$ near the anti-nodal regions best captures the observed enhancement in $R_{\mathrm{H}}(0)$ with reduced doping, in line with scenario $I$.

\begin{tabular}{|c|c|c|c|}
\hline doping $p$ & $R_{\mathrm{H}}^{\text {full }}(0)\left(\mathrm{mm}^{3} / \mathrm{C}\right)$ & $R_{\mathrm{H}}^{\text {meas }}(0)\left(\mathrm{mm}^{3} / \mathrm{C}\right)$ & $R_{\mathrm{H}}^{\text {trunc }}(0)$ \\
\hline 0.20 & 0.296 & $0.69 \pm 0.09$ & $0.482 \pm 0.067$ \\
\hline 0.21 & 0.283 & $0.65 \pm 0.07$ & $0.578 \pm 0.081$ \\
\hline 0.21 & 0.283 & $0.65 \pm 0.07$ & $0.456 \pm 0.065$ \\
\hline 0.22 & 0.269 & $0.58 \pm 0.07$ & $0.404 \pm 0.057$ \\
\hline 0.23 & 0.257 & $0.49 \pm 0.06$ & $0.354 \pm 0.047$ \\
\hline 0.24 & 0.249 & $0.34 \pm 0.04$ & $0.360 \pm 0.042$ \\
\hline 0.24 & 0.249 & $0.40 \pm 0.06$ & $0.296 \pm 0.037$ \\
\hline 0.25 & 0.237 & $0.35 \pm 0.02$ & $0.251 \pm 0.032$ \\
\hline 0.26 & 0.228 & $0.28 \pm 0.01$ & $0.224 \pm 0.025$ \\
\hline 0.27 & 0.217 & - & $0.215 \pm 0.007$ \\
\hline 0.275 & 0.205 & 0.22 & $0.204 \pm 0.006$ \\
\hline 0.3 & 0.172 & 0.21 & $0.168 \pm 0.006$ \\
\hline 0.32 & 0.170 & 0.19 & $0.166 \pm 0.006$ \\
\hline
\end{tabular}

Table 10: Measured Hall coefficient $R_{\mathrm{H}}^{\text {meas }}(0)$ in the zero-temperature limit (based on Table 77 for OD LSCO together with $R_{\mathrm{H}}^{\text {full }}(0)$ - the calculated Hall coefficient assuming a fully coherent FS, and $R_{\mathrm{H}}^{\text {trunc }}(0)$ - the calculated Hall coefficient for a truncated FS within scenario $I$ determined using $n_{c o h}=(1+p)-n_{s}(0)$ and the $n_{s}(0)$ values listed in Table 9 .

\begin{tabular}{|c|c|c|c|c|}
\hline doping $p$ & $n_{\text {coh }}$ & $n_{\mathrm{s}}(0)$ & $\gamma(0) / \gamma_{N}$ & $R_{\mathrm{H}}(0)\left(\mathrm{mm}^{3} / \mathrm{C}\right)$ \\
\hline 0.20 & 0.54 & 0.23 & 0.48 & -4.41 \\
\hline 0.21 & 0.54 & 0.24 & 0.48 & -4.43 \\
\hline 0.22 & 0.55 & 0.23 & 0.48 & -4.23 \\
\hline 0.23 & 0.57 & 0.19 & 0.49 & -3.97 \\
\hline 0.24 & 0.59 & 0.14 & 0.49 & -3.57 \\
\hline 0.26 & 0.63 & 0.00 & 0.50 & -2.82 \\
\hline
\end{tabular}

Table 11: Calculated $n_{\mathrm{s}}(0), n_{\text {coh }}$ and $R_{\mathrm{H}}(0)$ for scenario $C$ in which the incoherent carriers occupy the nodal regions of the Fermi surface. The large negative Hall coefficients are clearly inconsistent with the literature. Moreover, the lack of a strong doping dependence in $\gamma(0) / \gamma_{N}$ fails to account for the measured residual specific heat in OD LSCO.

\section{B.4 The weighting factor $f_{\sigma}$ in $\mathbf{L a}_{2-x} \mathrm{Sr}_{x} \mathrm{CuO}_{4}$}

As described in section A.1, in a model based on coherent and incoherent conductivity channels summing in parallel, $R_{\mathrm{H}}(0)$ has to be renormalised by the weighting factor $f_{\sigma}$ in order to obtain an estimate for the coherent carrier density $n_{c o h}$. The weighting factor $f_{\sigma}$ is equal to the square of the ratio between the zero-field conductivity of the coherent channel and the total zero-field conductivity (Eq. $(13)$ ) and can be determined by fitting the total zero-field resistivity, as described in section A.2 for T12201. For full consistency, therefore, the same weighting factor $f_{\sigma}$ should also be included when extracting the coherent carrier density from the measured Hall coefficient in LSCO. In contrast to Tl2201, however, it has proven impossible to perform a reliable 5-parameter fitting procedure on the zerofield resistivity in LSCO to extract $f_{\sigma}$. This difficulty likely stems from the fact that $\ell_{0}$ can be 1 to 2 orders of magnitude smaller at the zone boundary than along the zone 
diagonals, even if the entire FS of OD LSCO were coherent. Taking into account any additional scattering, e.g. on critical fluctuations, that could drive states at the zone edges incoherent, the contribution to $\sigma_{x x}$ from those states would become even smaller. In light of this, it would appear that the total conductivity in the zero-temperature limit comes almost entirely from the coherent sector, i.e. that $f_{\sigma} \approx 1$. Thus, in our analysis of $R_{\mathrm{H}}(0)$, we have assumed that $f_{\sigma}=1$.

Having established that $f_{\sigma} \approx 1$, a natural question arises: if the total conductivity is dominated by the coherent sector, why does the zero-field resistivity of OD LSCO vary almost linearly with temperature over such a wide doping range [12]? In order to address this question, we first recall that the total conductivity in LSCO is dominated by the coherent sector only in the zero-temperature limit, where the impurity scattering is not screened by other scattering mechanisms. With increasing temperature, when other scattering mechanisms come into play, it is expected that the coherent sector, whose resistivity grows faster as $T+T^{2}$ (see section A.2), is no longer dominating the total conductivity entirely. Such behavior can also be seen in Tl2201 (see Fig. 3) where, at low $T$, the coherent sector gives the dominant contribution, while at higher temperatures, the mixing between the two channels produces the total resistivity that shows an almost $T$-linear dependence on approaching $300 \mathrm{~K}$. In case of LSCO, such a mixing is expected to occur at much lower temperatures, meaning that the coherent sector grows much faster than in case of Tl2201, which, based on zero-field resistivity alone, is impossible to determine.

\section{B.5 Additional considerations for $\mathrm{La}_{2-x} \mathrm{Sr}_{x} \mathrm{CuO}_{4}$}

While the data plotted in Fig. 2 appears to show that the relation $n_{\text {coh }}+n_{s}(0)=1+p$ holds equally well in both OD Tl2201 and LSCO, we conclude this section by considering here other factors that have been ignored until now and that may influence the final robustness of the posited relation.

The first point of consideration is that all calculations of $\sigma_{x x}, \sigma_{x y}$ and $n_{s}(0)$ were performed for a strictly 2D FS. While this is likely to be a good approximation for OD T2201, where the resistivity anisotropy is more than 1000 [86], it is not immediately clear whether the approximation holds as well in OD LSCO, where the resistivity anisotropy becomes less than 50 [44]. It is not known at present how these calculations will be modified by inclusion of a finite $c$-axis FS warping, though we expect any modifications due to the warping to be averaged out in a full 3D integration.

The second point of consideration is the difference between single crystals and thin films. While the majority of the analysis has been performed on transport and thermodynamic data obtained on bulk single crystals, all $n_{s}(0)$ values were obtained from penetration depth measurements carried out on thin films. Strain from the substrate is known to modify the properties of LSCO, but it is not at all clear how to factor this into the calculations. In the work by Lemberger et al. [37], for example, two of their films with nominal $x$ values of 0.27 and 0.30 - are found to have $T_{c}$ values of $21 \mathrm{~K}$ and $9 \mathrm{~K}$. We did not use these samples in our analysis as their $T_{c}$ values are far from the expected (Presland) parabola 83 and their corresponding $1 / \lambda_{a b}^{2}(0)$ values markedly different from the values quoted by Božović et al [7]. At the same time, it is noted that superconductivity in the Božović films vanishes at a Sr concentration of 0.26 , while in single crystals, $p_{S C}=0.27$. Such a small shift in the range of superconductivity, however, will only modify the analysis slightly and is not expected to affect any of the main conclusions. 


\section{Dirty d-wave scenario in overdoped cuprates revisited}

Following the reports on the anomalous superfluid density [7] and optical conductivity 87 in OD LSCO, a dedicated theoretical study was carried out seeking to explain such behavior within a dirty $d$-wave scenario based on weak-coupling BCS theory 63 65]. Specifically, the effects of impurity scattering were taken into account through a self-consistent T-matrix approximation (SCTMA) and found to reproduce both the magnitude of the superfluid density and its (predominantly linear) dependence on temperature, provided that the vast majority of impurity scatterers were in the Born limit. In this section, we confirm the parameterization used in these calculations is consistent with that obtained from transport studies but show that the same theory fails to account for the apparent insensitivity of $T_{c}$ to the absolute value of the residual resistivity $\rho_{0}$ in OD cuprates.

The key parameter in the SCTMA analysis is $\Gamma_{N}(0)$, the zero-temperature normal state scattering rate. Disorder leads to a closing of the energy gap at a reduced $T_{c}$, the reduction being set by the celebrated Abrikosov-Gorkov formula [88]. Importantly, $T_{c}$ is found to depend only on $\Gamma_{N} / T_{c 0}$, while the form of $\rho_{s}(T)$ is influenced heavily by the impurity phase shift. Lee-Hone et al. 63] showed that within the Born limit, $\rho_{s}(T)$ remains $T$-linear at the lowest temperature even for $\Gamma_{N}(0)=0.5 T_{c 0}$, despite the fact that both $\rho_{s}(0)$ and $T_{c}$ have been reduced by approximately $40 \%$.

The scattering rate relevant for determining the drop in superfluid density is the elastic scattering rate set by the residual resistivity $\rho_{0}$. As done by Lee-Hone et al. 63, the resistivity is obtained from the Drude formula:

$$
\rho_{0}=\frac{1}{\sigma_{0}}=\frac{m^{*}}{n e^{2}} \Gamma_{t r}(0)
$$

where $m^{*}$ is the effective mass, $n$ is the carrier density and $\Gamma_{t r}(0)$ is the transport relaxation rate. The carrier density is estimated simply from the Sr content $x$ with $n=(1+x) / V_{\text {cell }}$. For $x=0.24$, we obtain $m^{*} \approx 8 m_{e}$ from the low- $T$ electronic specific heat 89 . For $\rho(0) \approx 16 \mu \Omega \mathrm{cm}$, one then obtains $\Gamma_{t r}(0) \approx \Gamma_{N}(0) \approx 55 \mathrm{~K}$, in agreement with Lee-Hone et al. 90. Taking into account the FS geometry, the anisotropy in the Fermi velocity and in the lifetime $\tau_{t r}(\phi)$ deduced from Hall effect measurements [34, produces only a $10 \%$ variation in this estimate of $\Gamma_{N}(0)$. Note, however, that this estimate of $\Gamma_{t r}(0)$ is a lower limit since the true value of $m^{*}$ in this formula is more likely to be closer to the unrenormalized or band mass value.

The key feature of Ref. 63 is the strong sensitivity of $T_{c}$ to the value of $\Gamma_{N}(0)$. LeeHone et al. found, for example, that for a $\Gamma_{N}(0)$ of this magnitude (more precisely, a residual resistivity of $16 \mu \Omega \mathrm{cm}$ ), the transition temperature of an OD LSCO film with $x=0.25$ is suppressed from its clean-limit value of $65 \mathrm{~K}$ to $10 \mathrm{~K}$. This strong sensitivity of $T_{c}$ to the value of $\Gamma_{N}(0)$ implies that samples with different residual resistivities should have markedly different $T_{c}$ (and $\rho_{s}$ ) values. Zn is known to have a strong detrimental effect on $T_{c}$ 91, other dopants less so. Recall that according to the SCTMA, the depression in $T_{c}$ should not depend on the strength of the scatterer nor on the value of the impurity phase shift. In Figure 8, we show resistivity data for a LSCO $x=0.26$ single crystal with a $T_{c}$ value of $5 \mathrm{~K}$, commensurate with its doping level. The residual resistivity of this sample is $\rho_{0}=50 \mu \Omega \mathrm{cm}$, giving a corresponding $\Gamma_{N}(0) \approx 160 \mathrm{~K}$. The latter appears far too high to sustain superconductivity within the dirty $d$-wave scenario.

One might argue, of course, that the doping level is actually shifted to lower doping, e.g. due to oxygen vacancies, leading to a sample that, were it cleaner, would have a much higher $T_{c}$. This argument does not hold, however, when one examines the $T$-dependence of $\rho_{a b}(T)$. As shown previously [92], $\rho_{a b}(T)$ below about $150 \mathrm{~K}$ can be approximated by 
the expression $\rho_{0}+\alpha_{1}(0) T+\alpha_{2} T^{2}$ across the entire strange metal regime of OD cuprates. Approaching room temperature, $\rho_{a b}(T)$ becomes $T$-linear again, but with a different hightemperature slope $\alpha_{1}(\infty) T\left[92\right.$. While $\alpha_{2}$ and $\alpha_{1}(\infty)$ are essentially doping-independent, $\alpha_{1}(0)$ is found to grow linearly from zero at $p 0.31$ to a maximum at $p^{*}=0.19[12$. This trend is found in all families of OD cuprates studied to date, including LSCO [12], Tl2201 $\left[13\right.$ and Bi2201 [14]. Thus, the magnitude of $\alpha_{1}(0)$ (more robustly, the ratio $\alpha_{1}(0) / \alpha_{1}(\infty)$ which removes any geometrical uncertainty as well as differences in the unit cell volume or FS topology between the different families) can provide a good gauge of the doping level of a particular sample [92]. For the sample shown in Fig. 8A, $\alpha_{1}(0) / \alpha_{1}(\infty)=0.4$, consistent with a doping of $p=0.26$. Hence, the above argument does not appear to hold.
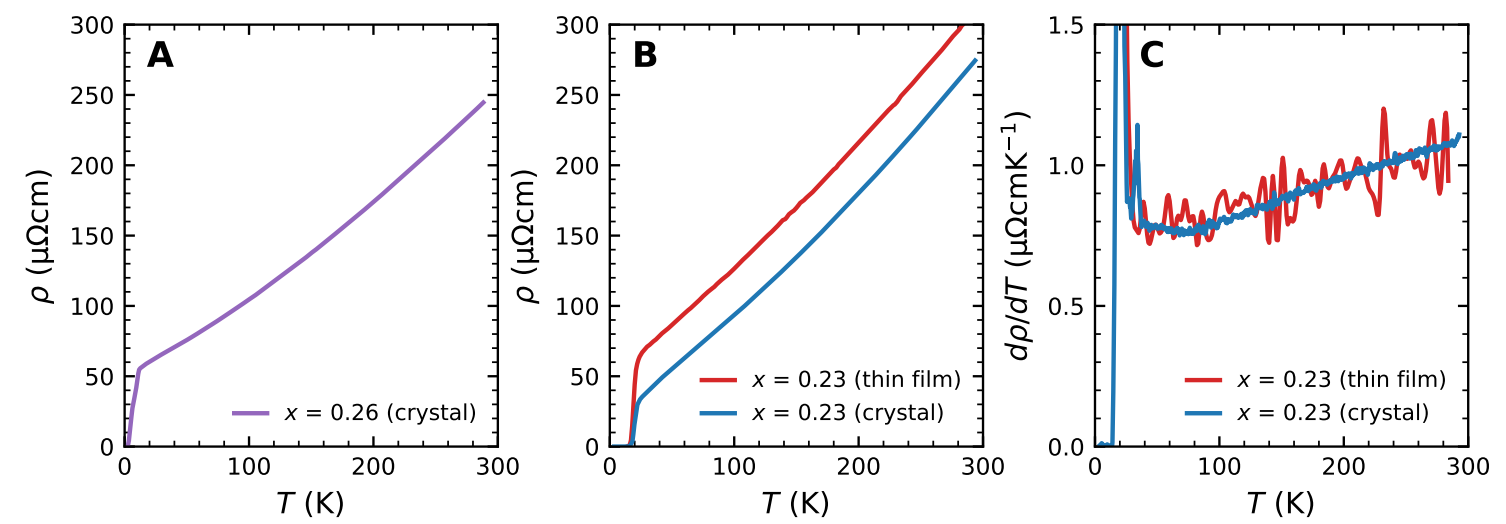

Figure 8: A): In-plane resistivity of LSCO26 single crystal $\left(T_{c} \approx 5 \mathrm{~K}\right)$. Note that the magnitude of the residual resistivity $\rho_{0}=50 \mu \Omega \mathrm{cm}$. B): Comparison of the in-plane resistivity of LSCO23 single crystal (blue curve) from Ref. 12 and a LSCO23 thin film (red curve) from Ref. [80]. The $\rho_{a b}(T)$ data for the thin film has been divided by 2 in order to normalize the slopes. The corresponding $\rho_{0}$ values are 20 and $50 \mu \Omega \mathrm{cm}$ respectively. C): Temperature derivatives $d \rho_{a b} / d T$ of the same (normalized) resistivity curves. The derivatives are identical within the scatter over the entire temperature range.

Fig. $8 \mathrm{~B}$ shows the resistivity curves of a LSCO single crystal [12] and a thin film [80] with nominally the same doping level $(x=0.23)$ and similar $T_{c}$ values $(19 \mathrm{~K}$ and $20 \mathrm{~K}$, as determined by the mid-point of their resistive transitions). Note that the resistivity curve of the thin film has been scaled by a factor of 0.5 in order to normalize the slopes of the two curves. Even after scaling, however, the residual resistivity of the film $\left(\rho_{0}=50 \mu \Omega \mathrm{cm}\right)$ is still 2.5 times larger than for the single crystal $\left(\rho_{0}=20 \mu \Omega \mathrm{cm}\right)$. Nevertheless, its $T_{c}$ value is almost identical. Moreover, the form of $\rho_{a b}(T)$ in both samples is the same, as shown by plotting the derivative in Fig. $8 \mathrm{C}$, confirming that their doping levels are essentially equivalent.

According to Lee-Hone et al., for $\Gamma_{N}(0) \approx 50 \mathrm{~K}$, the $T_{c}$ of LSCO23 would be reduced from $75 \mathrm{~K}$ to $25 \mathrm{~K}$. Hence, a shift in $\Gamma_{N}(0)$ from $65 \mathrm{~K}$ to $160 \mathrm{~K}$ would, according to the theory, effectively kill superconductivity outright. Yet the $T_{c}$ is not only the same in both samples, it is also consistent with the usual $T_{c}$ parabola [83]. This observation is inconsistent with expectations from the dirty $d$-wave scenario and thus raises an important challenge to the applicability of the SCTMA treatment of BCS theory to OD cuprates. 


\section{References}

[1] P. A. Lee and X.-G. Wen, Unusual superconducting state of underdoped cuprates, Phys. Rev. Lett. 78, 4111 (1997), doi:10.1103/PhysRevLett.78.4111.

[2] S. Hüfner, M. A. Hossain, A. Damascelli and G. A. Sawatzky, Two gaps make a high-temperature superconductor?, Rep. Prog. Phys. 71(6), 062501 (2008), doi:10.1088/0034-4885/71/6/062501.

[3] T. Valla, I. K. Drozdov and G. D. Gu, Disappearance of superconductivity due to vanishing coupling in the overdoped $\mathrm{Bi}_{2} \mathrm{Sr}_{2} \mathrm{CaCu}_{2} \mathrm{O}_{8+\delta}$, Nature Commun. 11(1), 569 (2020), doi:10.1038/s41467-020-14282-4.

[4] Y. J. Uemura, A. Keren, L. P. Le, G. M. Luke, W. D. Wu, Y. Kubo, T. Manako, Y. Shimakawa, R. Subramanian, J. L. Cobb and J. T. Markert, Magnetic-field penetration depth in $\mathrm{Tl}_{2} \mathrm{Ba}_{2} \mathrm{CuO}_{6+\delta}$ in the overdoped regime, Nature 364, 605 (1993), doi: $10.1038 / 364605 \mathrm{a} 0$.

[5] C. Niedermayer, C. Bernhard, U. Binninger, H. Glückler, J. L. Tallon, E. J. Ansaldo and J. I. Budnick, Muon spin rotation study of the correlation between $T_{c}$ and $n_{s} / m^{*}$ in overdoped $\mathrm{Tl}_{2} \mathrm{Ba}_{2} \mathrm{Cu} \mathrm{O}_{6+\delta}$, Phys. Rev. Lett. 71, 1764 (1993), doi:10.1103/PhysRevLett.71.1764.

[6] D. Deepwell, D. C. Peets, C. J. S. Truncik, N. C. Murphy, M. P. Kennett, W. A. Huttema, R. Liang, D. A. Bonn, W. N. Hardy and D. M. Broun, Microwave conductivity and superfluid density in strongly overdoped $\mathrm{Tl}_{2} \mathrm{Ba}_{2} \mathrm{CuO}_{6+\delta}$, Phys. Rev. B 88, 214509 (2013), doi:10.1103/PhysRevB.88.214509.

[7] I. Božović, X. He, J. Wu and A. T. Bollinger, Dependence of the critical temperature in overdoped copper oxides on superfluid density, Nature 536(7616), 309 (2016), doi:10.1038/nature19061.

[8] B. Vignolle, A. Carrington, R. A. Cooper, M. M. J. French, A. P. Mackenzie, C. Jaudet, D. Vignolles, C. Proust and N. E. Hussey, Quantum oscillations in an overdoped high-T $T_{c}$ superconductor, Nature 455(7215), 952 (2008), doi:10.1038/nature07323.

[9] B. Keimer, S. A. Kivelson, M. R. Norman, S. Uchida and J. Zaanen, From quantum matter to high-temperature superconductivity in copper oxides, Nature 518(7538), 179 (2015), doi:10.1038/nature14165.

[10] A. P. Mackenzie, S. R. Julian, D. C. Sinclair and C. T. Lin, Normal-state magnetotransport in superconducting $\mathrm{Tl}_{2} \mathrm{Ba}_{2} \mathrm{Cu} \mathrm{O}_{6+\delta}$ to millikelvin temperatures, Phys. Rev. B 53(9), 5848 (1996), doi:10.1103/PhysRevB.53.5848.

[11] C. Proust, E. Boaknin, R. W. Hill, L. Taillefer and A. P. Mackenzie, Heat transport in a strongly overdoped cuprate: Fermi-liquid and a pure d-Wave BCS superconductor, Phys. Rev. Lett. 89, 147003 (2002), doi:10.1103/PhysRevLett.89.147003.

[12] R. A. Cooper, Y. Wang, B. Vignolle, O. J. Lipscombe, S. M. Hayden, Y. Tanabe, T. Adachi, Y. Koike, M. Nohara, H. Takagi, C. Proust and N. E. Hussey, Anomalous criticality in the electrical resistivity of $\mathrm{La}_{2-x} \mathrm{Sr}_{x} \mathrm{CuO} \mathrm{O}_{4}$, Science 323(5914), 603 (2009), doi:10.1126/science.1165015. 
[13] N. E. Hussey, H. Gordon-Moys, J. Kokalj and R. H. McKenzie, Generic strangemetal behaviour of overdoped cuprates, J. Phys.: Conf. Ser. 449, 012004 (2013), doi:10.1088/1742-6596/449/1/012004.

[14] C. Putzke, S. Benhabib, W. Tabis, J. Ayres, Z. Wang, L. Malone, S. Licciardello, J. Lu, T. Kondo, T. Takeuchi, N. E. Hussey, J. R. Cooper et al., Reduced Hall carrier density in the overdoped strange metal regime of cuprate superconductors, arXiv:1909.08102 [cond-mat] (2019).

[15] P. Giraldo-Gallo, J. A. Galvis, Z. Stegen, K. A. Modic, F. F. Balakirev, J. B. Betts, X. Lian, C. Moir, S. C. Riggs, J. Wu, A. T. Bollinger, X. He et al., Scaleinvariant magnetoresistance in a cuprate superconductor, Science 361(6401), 479 (2018), doi:10.1126/science.aan3178.

[16] J. Ayres, M. Berben, M. Čulo, Y.-T. Hsu, E. van Heumen, Y. Huang, J. Zaanen, T. Kondo, T. Takeuchi, J. R. Cooper, C. Putzke, S. Friedemann et al., Magnetoresistance scaling in the strange metal regime of highly overdoped cuprates, arXiv:2012.01208 [cond-mat.str-el] (2020).

[17] I. M. Hayes, R. D. McDonald, N. P. Breznay, T. Helm, P. J. W. Moll, M. Wartenbe, A. Shekhter and J. G. Analytis, Scaling between magnetic field and temperature in the high-temperature superconductor $B a F e_{2}\left(A s_{1-x} P_{x}\right)_{2}$, Nature Phys. 12(10), 916 (2016), doi:10.1038/nphys3773.

[18] T. Sarkar, P. R. Mandal, N. R. Poniatowski, M. K. Chan and R. L. Greene, Correlation between scale-invariant normal-state resistivity and superconductivity in an electron-doped cuprate, Sci. Adv. 5(5), eaav6753 (2019), doi:10.1126/sciadv.aav6753.

[19] S. Licciardello, N. Maksimovic, J. Ayres, J. Buhot, M. Čulo, B. Bryant, S. Kasahara, Y. Matsuda, T. Shibauchi, V. Nagarajan, J. G. Analytis and N. E. Hussey, Coexistence of orbital and quantum critical magnetoresistance in $\mathrm{FeSe}_{1-x} S_{x}$, Phys. Rev. Res. 1(2), 023011 (2019), doi:10.1103/PhysRevResearch.1.023011.

[20] N. E. Hussey, J. Buhot and S. Licciardello, A tale of two metals: contrasting criticalities in the pnictides and hole-doped cuprates, Reports on Progress in Physics 81(5), 052501 (2018), doi:10.1088/1361-6633/aaa97c.

[21] J. M. Wade, J. W. Loram, K. A. Mirza, J. R. Cooper and J. L. Tallon, Electronic specific heat of $\mathrm{Tl}_{2} \mathrm{Ba}_{2} \mathrm{CuO}_{6+\delta}$ from $2 \mathrm{~K}$ to $300 \mathrm{~K}$ for $0 \leq \delta \leq 0.1$, J. Supercond. 7 , 261 (1994), doi:10.1007/BF00730408.

[22] N. E. Hussey, M. Abdel-Jawad, A. Carrington, A. P. Mackenzie and L. Balicas, A coherent three-dimensional Fermi surface in a high-transition-temperature superconductor, Nature 425(6960), 814 (2003), doi:10.1038/nature01981.

[23] M. Platé, J. D. F. Mottershead, I. S. Elfimov, D. C. Peets, R. Liang, D. A. Bonn, W. N. Hardy, S. Chiuzbaian, S. Falub, M. Shi, L. Patthey and A. Damascelli, Fermi surface and quasiparticle excitations of overdoped $\mathrm{Tl}_{2} \mathrm{Ba}_{2} \mathrm{CuO}_{6+\delta}$, Phys. Rev. Lett. 95, 077001 (2005), doi:10.1103/PhysRevLett.95.077001.

[24] A. J. Leggett, Theory of a superfluid Fermi liquid. I. General formalism and static properties, Phys. Rev. 140, A1869 (1965), doi:10.1103/PhysRev.140.A1869. 
[25] F. Gross, B. S. Chandrasekhar, D. Einzel, K. Andres, P. J. Hirschfeld, H. R. Ott, J. Beuers, Z. Fisk and J. L. Smith, Anomalous temperature dependence of the magnetic field penetration depth in superconducting $U_{B e_{13}}$, Z. Phys. B Cond. Matt. 64(2), 175 (1986), doi:10.1007/BF01303700.

[26] K. Hashimoto, K. Cho, T. Shibauchi, S. Kasahara, Y. Mizukami, R. Katsumata, Y. Tsuruhara, T. Terashima, H. Ikeda, M. A. Tanatar, H. Kitano, N. Salovich et al., A sharp peak of the zero-temperature penetration depth at optimal composition in $\mathrm{BaFe}_{2}\left(\mathrm{As}_{1-x} P_{x}\right)_{2}$, Science 336(6088), 1554 (2012), doi:10.1126/science.1219821.

[27] J. Zaanen, Superconducting electrons go missing, Nature 536, 282 (2016), doi: $10.1143 /$ JPSJ.69.1602.

[28] J. H. Brewer, S. L. Stubbs, R. Liang, D. A. Bonn, W. N. Hardy, W. A. MacFarlane and D. C. Peets, $T F-\mu+S R$ in overdoped $\mathrm{Tl}_{2} \mathrm{Ba}_{2} \mathrm{Cu} \mathrm{O}_{(6+\delta)}$ : simplicity still elusive, J. Phys.: Conf. Series 551, 012030 (2014), doi:10.1088/1742-6596/551/1/012030.

[29] A. F. Bangura, P. M. C. Rourke, T. M. Benseman, M. Matusiak, J. R. Cooper, N. E. Hussey and A. Carrington, Fermi surface and electronic homogeneity of the overdoped cuprate superconductor $\mathrm{Tl}_{2} \mathrm{Ba}_{2} \mathrm{CuO}_{6+\delta}$ as revealed by quantum oscillations, Phys. Rev. B 82(14), 140501 (2010), doi:10.1103/PhysRevB.82.140501.

[30] J. M. Wade, J. W. Loram, K. A. Mirza, J. R. Cooper and J. L. Tallon, Electronic specific heat of $\mathrm{Tl}_{2} \mathrm{Ba}_{2} \mathrm{CuO}_{(6+\delta)}$ from $2 \mathrm{~K}$ to $300 \mathrm{~K}$ for $0 \leq \delta \leq 0.1$., J. Supercond. 7, 261 (1994), doi:10.1007/BF00730408.

[31] J. M. Wade, Ph.D. thesis, University of Cambridge (The actual $\Delta \gamma(0)$ values are taken from Fig. 6.9 of the thesis, where entropy conservation has been ensured). (1995).

[32] P. M. C. Rourke, A. F. Bangura, T. M. Benseman, M. Matusiak, J. R. Cooper, A. Carrington and N. E. Hussey, A detailed de Haas-van Alphen effect study of the overdoped cuprate $\mathrm{Tl}_{2} \mathrm{Ba}_{2} \mathrm{CuO}_{6+\delta}$, New Journal of Physics 12(10), 105009 (2010), doi: $10.1088 / 1367-2630 / 12 / 10 / 105009$.

[33] T. Yoshida, X. J. Zhou, D. H. Lu, S. Komiya, Y. Ando, H. Eisaki, T. Kakeshita, S. Uchida, Z. Hussain, Z.-X. Shen and A. Fujimori, Low-energy electronic structure of the high-T $T_{c}$ cuprates $\mathrm{La}_{2-x} \mathrm{Sr}_{x} \mathrm{CuO}_{4}$ studied by angle-resolved photoemission spectroscopy., J. Phys.: Condens. Matter 19, 125209 (2007), doi:10.1088/0953$8984 / 19 / 12 / 125209$.

[34] A. Narduzzo, G. Albert, M. M. J. French, N. Mangkorntong, M. Nohara, H. Takagi and N. E. Hussey, Violation of the isotropic mean free path approximation for overdoped $\mathrm{La}_{2-x} \mathrm{Sr}_{x} \mathrm{CuO}$, Phys. Rev. B 77, 220502 (2008), doi:10.1103/PhysRevB.77.220502.

[35] Y. Fang, G. Grissonnanche, A. Legros, S. Verret, F. Laliberté, A. Ataei, M. Dion, D. Graf, M. J. Lawler, P. Goddard, L. Taillefer and B. J. Ramshaw, Fermi surface transformation at the pseudogap critical point of a cuprate superconductor., arXiV: 2004.01725 (2020).

[36] E. Abrahams and C. M. Varma, What angle-resolved photoemission experiments tell about the microscopic theory for high-temperature superconductors., Proc. Natl. Acad. Sci. U.S.A. 97, 5714 (2000), doi:10.1073/pnas.100118797. 
[37] T. R. Lemberger, I. Hetel, A. Tsukada and M. Naito, Anomalously sharp superconducting transitions in overdoped $\mathrm{La}_{2-x} \mathrm{Sr}_{x} \mathrm{CuO}_{4}$ films, Phys. Rev. B 82, 214513 (2010), doi:10.1103/PhysRevB.82.214513.

[38] Y. Wang, J. Yan, L. Shan, H.-H. Wen, Y. Tanabe, T. Adachi and Y. Koike, Weak-coupling d-wave BCS superconductivity and unpaired electrons in overdoped $\mathrm{La}_{2-x} \mathrm{Sr}_{x} \mathrm{CuO} \mathrm{O}_{4}$ single crystals, Phys. Rev. B 76, 064512 (2007), doi:10.1103/PhysRevB.76.064512.

[39] M. Nohara, H. Suzuki, M. Isshiki, N. Mangkorntong, F. Sakai and H. Takagi, Quasiparticle density of states of clean and dirty d-wave superconductors: mixed-state specific heat of $\mathrm{La}_{2-x} \mathrm{Sr}_{x} \mathrm{CuO}_{4}$ single crystals, J. Phys. Soc. Japan 69(6), 1602 (2000), doi:10.1143/JPSJ.69.1602

[40] S. Ohsugi, Y. Kitaoka, K. Ishida, G.-Q. Zheng and K. Asayama, Temperature dependence of spin susceptibility of $\mathrm{La}_{2-x} \mathrm{Sr}_{x} \mathrm{CuO} \mathrm{O}_{4} \mathrm{Cu}$ knight shift measurement, Physica C: Superconductivity 282-287, 1373 (1997), doi:10.1016/S0921-4534(97)00773-9.

[41] T. Kondo, Y. Nakashima, W. Malaeb, Y. Ishida, Y. Hamaya, T. Takeuchi and S. Shin, Anomalous doping variation of the nodal low-energy feature of superconducting $(\mathrm{Bi}, \mathrm{Pb})_{2}(\mathrm{Sr}, \mathrm{La})_{2} \mathrm{CuO}_{6+\delta}$ crystals revealed by laser-based angleresolved photoemission pectroscopy, Phys. Rev. Lett. 110, 217006 (2013), doi:10.1103/PhysRevLett.110.217006.

[42] N. Momono, M. Ido, T. Nakano, M. O. Oda, Y. Okajima and K. Yamaya, Low-T electronic specific heat of $\mathrm{La}_{2-x} \mathrm{Sr}_{x} \mathrm{CuO}_{4}$ and $\mathrm{La}_{2-x} \mathrm{Sr}_{x} \mathrm{Cu}_{1-y} \mathrm{Zn}_{y} \mathrm{O}_{4}$. Evidence for a $d$-wave superconductor, Physica 233C, 395 (1994), doi:10.1016/0921-4534(94)907684 .

[43] J. W. Loram, K. A. Mirza, J. R. Cooper and J. L. Tallon, Specific heat evidence on the normal state pseudogap, J. Phys. Chem Solids 59, 2091-2094 (1998), doi:10.1016/S0022-3697(98)00180-2.

[44] S. Nakamae, K. Behnia, N. Mangkorntong, M. Nohara, H. Takagi, S. J. C. Yates and N. E. Hussey, Electronic ground state of heavily overdoped nonsuperconducting $\mathrm{La}_{2-x} \mathrm{Sr}_{x} \mathrm{CuO}_{4}$, Phys. Rev. B 68, 100502(R) (2003), doi:10.1103/PhysRevB.68.100502.

[45] N. P. Ong, Geometric interpretation of the weak-field Hall conductivity in twodimensional metals with arbitrary Fermi surface, Phys. Rev. B 43, 193 (1991), doi:10.1103/PhysRevB.43.193.

[46] N. E. Hussey, The normal state scattering rate of high-T $T_{c}$ cuprates, Eur. Phys. J. B 31, 495 (2003), doi:10.1140/epjb/e2003-00059-9.

[47] J. E. Hirsch, Superconductivity from undressing, Phys. Rev. B 62, 14487 (2000), doi:10.1103/PhysRevB.62.14487.

[48] D. L. Feng, D. H. Lu, K. M. Shen, C. Kim, H. Eisaki, A. Damascelli, R. Yoshizaki, J.-i. Shimoyama, K. Kishio, G. D. Gu, S. Oh, A. Andrus et al., Signature of superfluid density in the single-particle excitation spectrum of $\mathrm{Bi}_{2} \mathrm{Sr}_{2} \mathrm{CaCu}_{2} \mathrm{O}_{8+\delta}$, Science 289(5477), 277 (2000), doi:10.1126/science.289.5477.277. 
[49] H. Ding, J. R. Engelbrecht, Z. Wang, J. C. Campuzano, S.-C. Wang, H.-B. Yang, R. Rogan, T. Takahashi, K. Kadowaki and D. G. Hinks, Coherent quasiparticle weight and its connection to high- $T_{c}$ superconductivity from angle-resolved photoemission, Phys. Rev. Lett. 87, 227001 (2001), doi:10.1103/PhysRevLett.87.227001.

[50] D. A. Bonn, P. Dosanjh, R. Liang and W. N. Hardy, Evidence for rapid suppression of quasiparticle scattering below $T_{c}$ in $\mathrm{YBa}_{2} \mathrm{Cu}_{3} \mathrm{O}_{7-\delta}$, Phys. Rev. Lett. 68, 2390 (1992), doi:10.1103/PhysRevLett.68.2390.

[51] K. Krishana, J. M. Harris and N. P. Ong, Quasiparticle mean free path in $\mathrm{YBa}_{2} \mathrm{Cu}_{3} \mathrm{O}_{7}$ measured by the thermal Hall conductivity, Phys. Rev. Lett. 75, 3529 (1995), doi:10.1103/PhysRevLett.75.3529.

[52] H. J. A. Molegraaf, C. Presura, D. van der Marel, P. H. Kes and M. Li, Superconductivity-induced transfer of in-plane spectral weight in $\mathrm{Bi}_{2} \mathrm{Sr}_{2} \mathrm{CaCu}_{2} \mathrm{O}_{8+\delta}$, Science 295(5563), 2239 (2002), doi:10.1126/science.1069947.

[53] A. F. Santander-Syro, R. P. S. M. Lobo, N. Bontemps, Z. Konstantinovic, Z. Z. Li and H. Raffy, Pairing in cuprates from high-energy electronic states, Europhys. Lett. 62(4), 568 (2003), doi:10.1209/epl/i2003-00388-9.

[54] G. Deutscher, A. F. Santander-Syro and N. Bontemps, Kinetic energy change with doping upon superfluid condensation in high-temperature superconductors, Phys. Rev. B 72, 092504 (2005), doi:10.1103/PhysRevB.72.092504.

[55] F. Carbone, A. B. Kuzmenko, H. J. A. Molegraaf, E. van Heumen, V. Lukovac, F. Marsiglio, D. van der Marel, K. Haule, G. Kotliar, H. Berger, S. Courjault, P. H. Kes et al., Doping dependence of the redistribution of optical spectral weight in $\mathrm{Bi}_{2} \mathrm{Sr}_{2} \mathrm{CaCu}_{2} \mathrm{O}_{8+\delta}$, Phys. Rev. B 74, 064510 (2006), doi:10.1103/PhysRevB.74.064510.

[56] J. Hwang, T. Timusk and G. D. Gu, Doping dependent optical properties of $\mathrm{Bi}_{2} \mathrm{Sr}_{2} \mathrm{CaCu}_{2} \mathrm{O}_{8+\delta}$, J. Phys.: Condens. Matt. 19(12), 125208 (2007), doi: $10.1088 / 0953-8984 / 19 / 12 / 125208$.

[57] F. Marsiglio, F. Carbone, A. B. Kuzmenko and D. van der Marel, Intraband optical spectral weight in the presence of a van Hove singularity: application to $\mathrm{Bi}_{2} \mathrm{Sr}_{2} \mathrm{CaCu}_{2} \mathrm{O}_{8+\delta}$, Phys. Rev. B 74, 174516 (2006), doi:10.1103/PhysRevB.74.174516.

[58] J. L. Tallon, J. W. Loram and C. Panagopoulos, Pseudogap and quantumtransition phenomenology in HTS cuprates, J. Low Temp. Phys. 131(3), 387 (2003), doi:10.1023/A:1022970312795.

[59] M. K. Chan, M. J. Veit, C. J. Dorow, Y. Ge, Y. Li, W. Tabis, Y. Tang, X. Zhao, N. Barišić and M. Greven, In-plane magnetoresistance obeys Kohler's rule in the pseudogap phase of cuprate superconductors, Phys. Rev. Lett. 113, 177005 (2014), doi:10.1103/PhysRevLett.113.177005.

[60] J. M. Harris, Y. F. Yan, P. Matl, N. P. Ong, P. W. Anderson, T. Kimura and K. Kitazawa, Violation of Kohler's rule in the normal-state magnetoresistance of $\mathrm{YBa}_{2} \mathrm{Cu}_{3} \mathrm{O}_{7-\delta}$ and $\mathrm{La}_{2-x} \mathrm{Sr}_{x} \mathrm{CuO}_{4}$, Phys. Rev. Lett. 75, 1391 (1995), doi:10.1103/PhysRevLett.75.1391. 
[61] S. Riggs, O. Vafek, J. B. Kemper, J. B. Betts, A. Migliori, F. F. Balakirev, W. N. Hardy, R. Liang, D. A. Bonn and G. S. Boebinger, Heat capacity through the magneticfield-induced resistive transition in an underdoped high-temperature superconductor, Nature Phys. 7, 332 (2011), doi:doi.org/10.1038/nphys1921.

[62] S. E. Sebastian, N. Harrison, F. F. Balakirev, M. M. Altarawneh, P. A. Goddard, R. Liang, D. A. Bonn, W. N. Hardy and G. G. Lonzarich, Normal-state nodal electronic structure in underdoped high-T $T_{c}$ copper oxides, Nature 511(7507), 61 (2014), doi: $10.1038 /$ nature13326.

[63] N. R. Lee-Hone, J. S. Dodge and D. M. Broun, Disorder and superfluid density in overdoped cuprate superconductors, Phys. Rev. B 96, 024501 (2017), doi:10.1103/PhysRevB.96.024501.

[64] N. R. Lee-Hone, V. Mishra, D. M. Broun and P. J. Hirschfeld, Optical conductivity of overdoped cuprate superconductors: application to $\mathrm{La}_{2-x} \mathrm{Sr}_{x} \mathrm{CuO} \mathrm{O}_{4}$, Phys. Rev. B 98, 054506 (2018), doi 10.1103/PhysRevB.98.054506.

[65] N. R. Lee-Hone, H. U. Özdemir, V. Mishra, D. M. Broun and P. J. Hirschfeld, Low energy phenomenology of the overdoped cuprates: viability of the Landau-BCS paradigm, Phys. Rev. Res. 2, 013228 (2020), doi:10.1103/PhysRevResearch.2.013228.

[66] D. Pelc, P. Popčević, M. Greven and N. Barišić, Universal behavior of cuprates explained by heterogeneous charge localization, Science Advances 5, eaau4538 (2019), doi:10.1126/sciadv.aau4538.

[67] M. Abdel-Jawad, M. P. Kennett, L. Balicas, A. Carrington, A. P. Mackenzie, R. H. McKenzie and N. E. Hussey, Anisotropic scattering and anomalous normal-state transport in a high-temperature superconductor, Nature Physics 2(12), 821 (2006), doi: $10.1038 /$ nphys449.

[68] J. A. N. Bruin, H. Sakai, R. S. Perry and A. P. Mackenzie, Similarity of scattering rates in metals showing T-linear resistivity., Science 339(6121), 804 (2013), doi:10.1126/science.1227612.

[69] A. Legros, S. Benhabib, W. Tabis, F. Laliberté, M. Dion, M. Lizaire, B. Vignolle, D. Vignolles, H. Raffy, Z. Z. Li, P. Auban-Senzier, N. Doiron-Leyraud et al., Universal T-linear resistivity and Planckian dissipation in overdoped cuprates., Nature Physics 15, 142 (2019), doi:10.1038/s41567-018-0334-2.

[70] S. Licciardello, J. Buhot, J. Lu, J. Ayres, S. Kasahara, Y. Matsuda, T. Shibauchi and N. E. Hussey, Electrical resistivity across a nematic quantum critical point, Nature 567(7747), 213 (2019), doi:10.1038/s41586-019-0923-y.

[71] J. Chang, M. Månsson, S. Pailhès, T. Claesson, O. J. Lipscombe, S. M. Hayden, L. Patthey, O. Tjernberg and J. Mesot, Anisotropic breakdown of Fermi liquid quasiparticle excitations in overdoped $\mathrm{La}_{2-x} \mathrm{Sr}_{x} \mathrm{CuO}_{4}$, Nature Communications 4, 2559 (2013), doi:10.1038/ncomms3559.

[72] R.-H. He, M. Fujita, M. Enoki, M. Hashimoto, S. Iikubo, S.-K. Mo, H. Yao, T. Adachi, Y. Koike, Z. Hussain, Z.-X. Shen and K. Yamada, Hidden Itinerant-Spin Phase in Heavily Overdoped $\mathrm{La}_{2-x} \mathrm{Sr}_{x} \mathrm{CuO} \mathrm{O}_{4}$ Superconductors Revealed by Dilute Fe Doping: A Combined Neutron Scattering and Angle-Resolved Photoemission Study, Phys. Rev. Lett. 107, 127002 (2011), doi:10.1103/PhysRevLett.107.127002. 
[73] C.-K. Kim, I. K. Drozdov, K. Fujita, J. C. Davis, I. Božović and T. Valla, Insitu angle-resolved photoemission spectroscopy of copper-oxide thin films synthesized by molecular beam epitaxy., J. El. Spect. Rel. Phen. pp. 0368-2048 (2018), doi: $10.1016 /$ j.elspec.2018.07.003.

[74] S. R. Park, Y. Cao, Q. Wang, M. Fujita, K. Yamada, S.-K. Mo, D. S. Dessau and D. Reznik, Broken relationship between superconducting pairing interaction and electronic dispersion kinks in $\mathrm{La}_{2-x} \mathrm{Sr}_{x} \mathrm{CuO}_{4}$ measured by angle-resolved photoemission., Phys. Rev. B 88, 220503 (2013), doi:10.1103/PhysRevB.88.220503.

[75] T. Nishikawa, J. Takeda and M. Sato, Transport anomalies of high- $T_{c}$ oxides above room temperature, J. Phys. Soc. Japan 63, 1441 (1994), doi:10.1143/JPSJ.63.1441.

[76] H. Y. Hwang, B. Batlogg, H. Takagi, H. L. Kao, J. Kwo, R. J. Cava, J. J. Krajewski and W. F. J. Peck, Scaling of the temperature dependent Hall effect in $\mathrm{La}_{2-x} \mathrm{Sr}_{x} \mathrm{CuO}_{4}$, Phys. Rev. Lett. 72, 2636 (1994), doi:10.1103/PhysRevLett.72.2636.

[77] F. F. Balakirev, J. B. Betts, A. Migliori, I. Tsukada, Y. Ando and G. S. Boebinger, Quantum phase transition in the magnetic-field-induced normal state of optimumdoped high- $T_{c}$ cuprate superconductors at low temperatures., Phys. Rev. Lett. 102, 017004 (2009), doi:10.1103/PhysRevB.75.024515.

[78] S. Ono, S. Komiya and Y. Ando, Strong charge fluctuations manifested in the hightemperature Hall coefficient of high-T $T_{c}$ cuprates, Phys. Rev. B 75, 024515 (2007), doi:10.1103/PhysRevLett.102.017004.

[79] I. Tsukada and S. Ono, Negative Hall coefficients of heavily overdoped $\mathrm{La}_{2-x} \mathrm{Sr}_{x} \mathrm{CuO}_{4}$, Phys. Rev. B 74, 134508 (2006), doi:10.1103/PhysRevB.74.134508

[80] Y. Ando, G. S. Boebinger, A. Passner, N. L. Wang, C. Geibel, F. Steglich, I. E. Trofimov and F. F. Balakirev, Normal-state Hall effect and the insulating resistivity of high- $T_{c}$ cuprates at low temperatures, Phys. Rev. B 56, R8530 (1997), doi:10.1103/PhysRevB.56.R8530.

[81] Y. Ando, Y. Kurita, S. Komiya, S. Ono and K. Segawa, Evolution of the Hall coefficient and the peculiar electronic structure of the cuprate superconductors, Phys. Rev. Lett. 92, 197001 (2004), doi:10.1103/PhysRevLett.92.197001.

[82] R. Daou, N. Doiron-Leyraud, D. LeBoeuf, S. Y. Li, F. Laliberté, O. Cyr-Choinière, Y. J. Jo, L. Balicas, J.-Q. Yan, J.-S. Zhou, J. B. Goodenough and L. Taillefer, Linear temperature dependence of resistivity and change in the Fermi surface at the pseudogap critical point of a high-T $T_{c}$ superconductor, Nature Phys. 5, 31 (2009), doi: $10.1038 /$ nphys1109.

[83] M. R. Presland, J. L. Tallon, R. G. Buckley, R. S. Liu and N. E. Flower, General trends in oxygen stoichiometry effects on $T_{c}$ in Bi and Tl superconductors, Physica C 176(1), 95 (1991), doi:10.1016/0921-4534(91)90700-9.

[84] J. Loram, K. Mirza, J. Wade, J. Cooper and W. Liang, The electronic specific heat of cuprate superconductors, Physica C: Superconductivity 235-240, 134 (1994), doi:https://doi.org/10.1016/0921-4534(94)91331-5.

[85] D. Pelc, M. Vučković, M. S. Grbić, M. Požek, G. Yu, T. Sasagawa, M. Greven and N. Barišić, Emergence of superconductivity in the cuprates via a universal percolation process, Nature Communications 9(1), 4327 (2018), doi:10.1038/s41467-018-06707-y. 
[86] N. E. Hussey, J. R. Cooper, J. M. Wheatley, I. R. Fisher, A. Carrington, A. P. Mackenzie, C. T. Lin and O. Milat, Angular dependence of the c-axis normal state magnetoresistance in single crystal $\mathrm{Tl}_{2} \mathrm{Ba}_{2} \mathrm{CuO}_{6+\delta}$, Physical Review Letters 76(1), 122 (1996), doi:10.1103/PhysRevLett.76.122.

[87] F. Mahmood, X. He, I. Božović and N. P. Armitage, Locating the missing superconducting electrons in the overdoped cuprates $\mathrm{La}_{2-x} \mathrm{Sr}_{x} \mathrm{CuO}_{4}$, Phys. Rev. Lett. 122, 027003 (2019), doi:10.1103/PhysRevLett.122.027003.

[88] A. A. Abrikosov and L. P. Gor'kov, Contribution to the theory of superconducting alloys with paramagnetic impurities, Zh. Eksp. Teor. Fiz. 39 (1960), [Sov. Phys. JETP 12, 1243 (1961)].

[89] N. Momono, T. Matsuzaki, M. Oda and M. Ido, Superconducting condensation energy and pseudogap formation in $\mathrm{La}_{2-x} \mathrm{Sr}_{x} \mathrm{CuO}_{4}$ : new energy scale for superconductivity., J. Phys. Soc. Japan 71, 2832 (2002), doi:10.1143/JPSJ.71.2832.

[90] N. R. Lee-Hone, J. S. Dodge and D. M. Broun, Erratum: Disorder and superfluid density in overdoped cuprate superconductors. Phys. Rev. B 97, 219903E (2018). In this Erratum, it is remarked that all scattering parameters used in the actual calculations were a factor of $\pi$ larger than stated in the original paper. This is correct for all parameters except the residual resistivity $\rho_{0}$, which needs to retain the original value $16 \mu \Omega c m$, as quoted in Ref. [87] .

[91] Y. Fukuzumi, K. Mizuhashi, K. Takenaka and S. Uchida, Universal superconductorinsulator transition and $T_{c}$ depression in $\mathrm{Zn}$-substituted high- $T_{c}$ cuprates in the underdoped regime, Phys. Rev. Lett. 76, 684 (1996), doi:10.1103/PhysRevLett.76.684.

[92] N. E. Hussey, R. A. Cooper, X. Xu, Y. Wang, I. Mouzopoulou, B. Vignolle and C. Proust, Dichotomy in the T-linear resistivity in hole-doped cuprates 369(1941), 1626 (2011), doi:10.1098/rsta.2010.0196. 